\title{
Phytochemical composition and antimicrobial activity of Ochna thomasiana
}

\author{
MBITHI JUSTUS MUEMA ${ }^{1}$, ALEX K. MACHOCHO ${ }^{2, \boldsymbol{v}}$, NICHOLAS K. GIKONYO ${ }^{1}$ \\ ${ }^{1}$ Department of Pharmacy and Complementary/Alternative Medicine, Kenyatta University. Mfangano Island, Kakamega Centre, Suba North, Kenya \\ ${ }^{2}$ Department of Chemistry, Kenyatta University. Mfangano Island, Kakamega Centre, Suba North, Kenya. "email: machocho.alex@ku.ac.ke
}

Manuscript received: 9 July 2019. Revision accepted: 17 February 2020.

\begin{abstract}
Muema MJ, Machocho AK, Gikonyo NK. 2020. Phytochemical composition and antimicrobial activity of Ochna thomasiana. Biofarmasi J Nat Prod Biochem 18: 29-41. Infectious diseases are the leading cause of mortality worldwide despite the vigorous campaigns that have been made to combat them. This study aimed to determine and evaluate the biological activities of Ochna thomasiana Engl. \& Gilg ex Gilg. Here, we screened the plant extracts and tested them for their antibacterial activity against Salmonella typhi (clinical isolate), Pseudomonas aeruginosa, Escherichia coli, Bacillus subtilis, and Staphylococcus aureus. Various chromatographic techniques separated and isolated the active compounds from this plant. The extracts were purified using silica gel, column chromatography (CC), Sephadex gel, and preparative thin-layer chromatography (PTLC). Structure characterization was determined using standard spectroscopic methods: Infrared (IR), ultraviolet (UV) spectroscopy, mass spectroscopy (MS) and proton nuclear magnetic resonance $\left({ }^{1} \mathrm{H}\right.$ NMR), distortionless enhancement by polarization transfer (DEPT), carbon-13 nuclear magnetic resonance (13C NMR), coherence spectroscopy (COSY), heteronuclear multiple bond coherence (HMBC) and heteronuclear single quantum coherence (HSQC). Compounds identified from the extracts are Lophirone A (18), afzelone D dimethyl ether (20), calodenone (17), a mixture of stigmasterol (74), and $\beta$-sitosterol (23) and 3 $\beta$-acetyl-24-ethylfriedelane (75). Methanol crude extracts' stem and root bark showed high activity against the Gram-positive bacteria. Lophirone A, afzelone D dimethyl ether, and 3 $\beta$-acetyl-24- ethylfriedelane showed high activity against $S$. aureus. The results showed that the root of O. thomasiana contains biflavonoids. Some sterols as their constituents and antimicrobial activity are significant and lead to the development of antimicrobial agents. The essential bioactive compounds and the antimicrobial activity of the crude extracts of this plant confirms its use in traditional medicine. However, there is a need to test the effectiveness of the crude extracts and isolated compounds via in vivo and in vitro evaluation. The plant species should be cultivated using good agricultural practices for medicinal plants for the future assessment of their activity against pathogens.
\end{abstract}

Keywords: Antimicrobial, Ochna thomasiana, phytochemistry

\section{INTRODUCTION}

The use of plants in indigenous cultures is multiple and very diverse. They form an important economic basis and consume as food, medicine, construction material, dyes, firewood, ritual paraphernalia, and ornaments. Plants have been the foundation of traditional medicine systems for thousands of years, where the knowledge of the plants has been passed on from one generation to another generation (Koehn and Carter 2005). The abundance of plants on the earth's surface has increased interest in investigating distinct extracts obtained from traditional medicinal plants as potential sources of new antimicrobial agents (Bonjar and Farrokhi 2004). Because people have used plants for millennia, much information on plants' therapeutic uses has accumulated, especially in the tropical parts of the world where plant diversity is much higher. In African, Chinese, and Indian communities, plants have become the main ingredient of traditional medicines (Gurib- Fakim 2006; Magassouba et al. 2007).

Medicinal plants are gifts of nature to cure a limitless number of diseases among human beings (Bushra and Ganga 2003). More than $66 \%$ of the population residing in developing countries was predicted by WHO (2008) to be depending directly on plants for their primary medical requirements (WHO 2008). This phenomenon is attributed to the fact that plant-derived medicines are readily accessible and relatively cheaper than pharmaceutical drugs (Amin and Mousa 2007; Ramawat and Goyal 2008; WHO 2008). Even the society of the developed world relies on their health care for plants directly or indirectly. In the United States, community pharmacies prescribe $11 \%$ of medicine consisting of plant extracts or active ingredients of plant origin (Cragg and Newman 2005). As many as $21 \%$ of patients in Dar es Salaam, Tanzania, who visited public hospitals had consulted a traditional healer before going to the hospital (de Boer et al. 2005). Medicine derived from the plant is taken in the form of teas, tinctures, poultices, and powders, depending on the knowledge of the use and application method (Fennell et al. 2004; Balunas and Kinghorn 2005).

Plants produce diverse compounds as a means of defense against fungi, bacteria, pests, as well as predators. Therefore, plants are efficient natural chemical factories, creating various structures of compounds that result in different physiological effects in the body upon ingestion (Edeoga et al. 2005). Substances isolated from plants were utilized as important drugs in one or more countries. Moreover, $60 \%$ of these substances were identified as a 
result of phytochemical studies on plants used for medicinal purposes (Farnsworth et al. 1985).

The natural products of the plant kingdom, primarily from higher plants may serve as a candidate for a new source of antimicrobial agents with possibly novel mechanisms of action (Shahidi 2004; Runyoro et al. 2006). The provision of safe and effective medicines might become a valuable tool to improve access to health care (WHO 2002; Duraipandiyan and Ignacimuthu 2007). Consequently, this has necessitated the investigation of plants as other potential sources of effective, safe, and cheap antimicrobial drugs (Thangadurai et al. 2004).

The objectives of this research were (i) To evaluate antimicrobial activities of the EtOAc, $\mathrm{MeOH}$, and DCM of crude extracts of $O$. Thomasiana, (ii) To elucidate the structures of the isolated compounds from the acquired spectrum using spectroscopic techniques, (iii) To evaluate antimicrobial activities of isolated pure compounds from $O$. thomasiana.

\section{MATERIALS AND METHODS}

\section{Plant collection and processing}

Plant samples (root and stem barks) were acquired from Arabuko-Sokoke, a forest in Malindi district, Kilifi County of Kenyas' coast province. The wood is located about 120 Km north of Mombasa town and transverses Kilifi County (ASFMT 2002). The plant identity was authenticated by a taxonomist of Kenyatta University, School of Medicine, Department of Pharmacy and Complementary/Alternative Medicine (Mr. Lucas Karimi) and confirmed at the herbarium of the Kenya National Museums where the Voucher specimen (number MM/OH/001/06) was submitted at Kenyatta University Botany Herbarium. The samples were air-dried for two weeks in the shade at room temperature $\left(25^{\circ} \mathrm{C} \pm 3^{\circ} \mathrm{C}\right)$ away from direct sunlight. The dry material was ground into a fine powder using a KHD Humboldt Wedag AG Electric grinding mill and then weighed.

\section{Reagents}

All reagents were purchased from Kobian, Nairobi, Kenya. Organic solvents used in this study include acetone, n-hexane, dichloromethane, ethyl acetate, and methanol. All solutions used for the extraction, fractionation, and crystallization were distilled two times before use and kept in amber bottles.

\section{Sequential extraction of root and stem barks}

The extraction protocol was based on the National Cancer Institute (NCI) protocol extraction of bioactive molecules (McCloud et al. 1998). Briefly, powdered material of $O$. thomasiana stem barks $(6.43 \mathrm{~kg})$ were soaked in 10 liters DCM for three days. The solvent must be sufficient enough to saturate plant material for thorough extraction thoroughly. Sequential extraction was done repeatedly with solvents of increasing polarity, starting with 8 liters ethyl acetate (EtOAc) and finally 7 liters methanol $(\mathrm{MeOH})$ for three days, each with occasional swirling to ensure thorough extraction. Similarly, the sequential extraction of $4.01 \mathrm{~kg}$ of powdered material $(O$. thomasiana root barks) was done using 8 liters DCM, 7 liters EtOAc, and finally 6 liters $\mathrm{MeOH}$ for three days each.

The extracts were filtered using a Buchner funnel using reduced pressure of a vacuum pump through filter paper (40 mm Whatman), the residue was soaked in solvent again for three days. The extraction resulted in a clear extract after three times of repetition. In each step, plant residue (material) was allowed to dry before appliying the next solvent. All the combined filtrates were concentrated using a rotary evaporator under reduced pressure at a temperature of $45{ }^{\circ} \mathrm{C}$ to recover back solvents. The crude extracts were kept in the desiccator to evaporate the excess solution and after that wrapped in aluminum foil and stored in the deep freezer to avoid decomposition of compounds. A portion of each crude extract ( $2 \mathrm{~g}$ ) was used for bioassay.

\section{Thin-layer chromatography (TLC)}

Analytical TLC was done on a $0.25 \mathrm{~mm}$ thickness of Silica gel $60 \mathrm{~F}_{254}$ (Macherey-Nagel) plates. The spots were visualized on the chromatograms using a multi-band ultraviolet light $(254 / 365 \mathrm{~nm})$ lamp (UV GL-58). The TLC plates were sprayed with $p$ - anisaldehyde solution and stored in the oven at $110^{\circ} \mathrm{C}$ until the spots appeared. Anisaldehyde was used as a developing reagent. The reagent contains a solution of $0.5 \mathrm{~mL} p$-anisaldehyde combined with $10 \mathrm{~mL}$ glacial acetic acid $85 \mathrm{~mL}$, ice-cold methanol or ethanol, and 98\% sulphuric acid (Randerath et al. 1968). Plants extracts were tested for content of the steroids/terpenes and flavonoids. The TLC plates were sprayed with the $p$-anisaldehyde solution and kept in the oven at $110^{\circ} \mathrm{C}$ until the spots appeared. Visualization of spots on developed TLC plates was done using long and short wavelengths $(\lambda)$ (365 and $254 \mathrm{~nm}$ respectively), on an ENF-240 C/F UV lamp (Spectronics Co., Westbury, UK).

\section{Melting point}

Uncorrected melting points were documented using open capillary tubes using a Gallenkamp melting point apparatus (Sanyo, West Sussex, and the UK).

\section{Infrared (IR) spectroscopy}

IR spectra were measured on an FTIR 8400S (Shimadzu, Japan). The spectra were calculated using potassium bromide $(\mathrm{KBr})$ pellets in the Chemistry research laboratory of Jomo Kenyatta University of Agriculture and Technology (JKUAT). A portion of the sample $(2 \mathrm{mg}$ ) was added and ground with $\mathrm{KBr}(100 \mathrm{mg})$ in a mortar to a fine powder until the sample was uniformly mixed. Then the mixture was pressed in a vacuum die to produce a transparent disk (pellet). The sample was later placed in a sample holder that was inserted in the spectrophotometer.

\section{Ultraviolet (UV) spectroscopy}

The UV spectra were determined on an ENF-240 C/F UV lamp (Spectronics Co., UK) in the Kenyatta University research laboratory instrument room. A tiny amount of the sample was dissolved in $\mathrm{MeOH}$ and chloroform, for only those samples that were soluble in them. This spectroscopic 
technique is ratio recording; where the ratio between the reference beam and the sample beam intensities $\left(I_{0} / I\right)$ was fed to a pen recorder. The recorder trace is set absorbance $(A)$ invariably against wavelength $(\lambda)$.

\section{Nuclear magnetic resonance (NMR) spectroscopy}

${ }^{1} \mathrm{H}(1 \mathrm{D}, 2 \mathrm{D} \mathrm{COSY})$ and ${ }^{13} \mathrm{C}$ spectra were recorded in $\mathrm{CDCl}_{3}$ and $\mathrm{CD}_{3} \mathrm{OD}$ as solvent with Tetramethylsilane (TMS) as internal standard on a Bruker $(400 \mathrm{MHz}$ and 600 $\mathrm{MHz})$ spectrometer operating at 400 and $600 \mathrm{MHz}\left({ }^{1} \mathrm{H}-\right.$ NMR), 100 and $150 \mathrm{MHz}\left({ }^{13} \mathrm{C}\right.$ NMR) machine at Kwazulu Natal University, South Africa and Nagasaki University, Japan Chemistry research laboratories, respectively. The peaks on ${ }^{1} \mathrm{H}$ NMR were determined as singlet $(s)$, doublet $(d)$, doublet of doublet $(d d)$, triplet $(t)$, quartet $(q)$, multiplet $(m)$ and (or) broad $(b)$ by employing TMS as reference. DEPT experiments determined the ${ }^{13} \mathrm{C}$ NMR multiplicity that gave chemical shift values for assignment. Chemical shifts were calculated in $\delta$ (ppm) and coupling constants, $J$, in hertz $(\mathrm{Hz})$. The sample was dissolved in $\mathrm{CD}_{3} \mathrm{OD}$ in a 5 mm NMR tube and stirred thoroughly. The solution was then transferred into an NMR tube and the spectrum recorded.

Two-dimensional NMR techniques may be utilized to access the correlations between nuclear in a compound. $J$ resolved 2D NMR experiments are useful in the measurement of ${ }^{13} \mathrm{C}^{-1} \mathrm{H}$ coupling in complex spin systems because they can separate overlapping multiplets. Correlated 2D tests applied to homonuclear spin systems include COSY (Correlated spectroscopy) while NOESY (Nuclear overhauser enhancement spectroscopy) is applied in the analysis of NMR spectra of large molecules (Abraham et al. 2003). HETCOR and HMQC displayed all the protons to which carbon atoms are attached although HMQC has higher sensitivity. On one axis the ${ }^{1} \mathrm{H}$ NMR spectrum is displayed while the other axis has the ${ }^{13} \mathrm{C}$ NMR spectrum, and thereby, signals indicate a direct coupling of the protons with the carbons (Silverstein et al. 2005). HMBC (Heteronuclear Multiple Bond Correlation) is a long-range correlation experiment which gives information about carbons bonded to proton which are 2-3 bonds away. HMBC has applications in every field for identification and characterization of the structure of compound (Vasavi et al. 2011).

\section{Mass Spectroscopy (MS)}

Mass spectrometers use the ratio difference in mass to charge $(\mathrm{m} / \mathrm{z})$ of ionized atoms or molecules to separate them. Mass spectroscopy allows the quantization of atoms or molecules and provides structural information by the identification of different fragmentation patterns (Uggerud et al. 2003). The analysis was done out at Chemistry research laboratory of Jomo Kenyatta University of Agriculture and Technology (JKUAT) using Shimadzu Gas Chromatography-Mass Spectrometry (GCMS). A small amount of a compound, approximately one micromole/ microgram or less was injected in the spectrometer and evaporated with vapor leaking into the ionization chamber set at a given pressure of about 10-7 mb.

\section{Antibacterial tests}

\section{Bacterial strains tests}

Plants extracts were screened in Mycology laboratory center for microbiology research at Kenya Medical Research Institute (KEMRI) against two standard strains of bacteria and two local clinical isolates from patients. The standard strains were obtained from the American Type Culture Collection (ATCC). The strains used were collected from Kenya National Public Health Laboratories (KNPHL), (KEMRI) and Plant and Microbial laboratory of Kenyatta University. Strains that were used included: Two Gram-negative bacteria, Pseudomonas aeruginosa (ATCC 27853), a reference strain, Salmonella typhi (Type K [I]) clinical isolate was also used. Also, two Gram-positive bacteria, Staphylococcus aureus, (ATCC 20572), a reference strain, and Bacillus subtilis (Type K [11]), clinical isolate. These microorganisms were cultivated in diagnostic sensitivity test agar (DST) and Muller-Hinton broth (MHB-Merck Germany).

\section{Preparation of media}

DST agar was according to the manufacturer instruction and dissolved in the recommended quantities of distilled water. MHB was made by using $28 \mathrm{~g}$ of the media, dissolved in 1 liter of distilled water and boiled. The media were sterilized separately by autoclaving at 15 atmospheres pressures and $121^{\circ} \mathrm{C}$ for 15 minutes, before used.

\section{Screening procedure}

The disc diffusion method was used for the initial activity of the tests on the plant extracts at concentrations of $450 \mu \mathrm{g} / \mathrm{disc}$. Concentrations of sample solutions were made by dissolving $1 \mathrm{~g}$ of extract in $1 \mathrm{~mL}$ of the solvent that had been used in their extractions, then followed by appropriate dilutions to the required concentrations. The resulting solution was then used to prepare the discs by the soaking method. Each disc was inoculated with of bacterial $(0.1 \mathrm{~mL})$ and yeast culture directly from the $24 \mathrm{~h}$ broth culture which was diluted to match 0.5 and 1.0 MacFarlands standard, respectively $\left(10^{8}\right.$ Colony Forming Units $(\mathrm{CFU}) / \mathrm{mL})$ and fungi diluted to match 1.0 MacFarland standard $\left(10^{8}\right.$ spores $\left./ \mathrm{mL}\right)$. The discs loaded with the extracts were then put onto the seeded plates (Figure 1).

The bacterial and yeast cultures were incubated for 24 and 48 hours, respectively at $37{ }^{\circ} \mathrm{C}$, while fungi were incubated at $25^{\circ} \mathrm{C}$ for five days. The zones of inhibition were measured and recorded in $\mathrm{mm}$, as described earlier (Elgayyar et al. 2000). Negative control plates were discs with sterile distilled water and methanol. Growth of the organism and sterility of media was controlled by use of broth only in negative control tubes, while broth plus microorganism in question in positive control tubes. Tetracycline served as the standard for positive control. All the controls were maintained at the same conditions as the tested samples (Figure 2). 


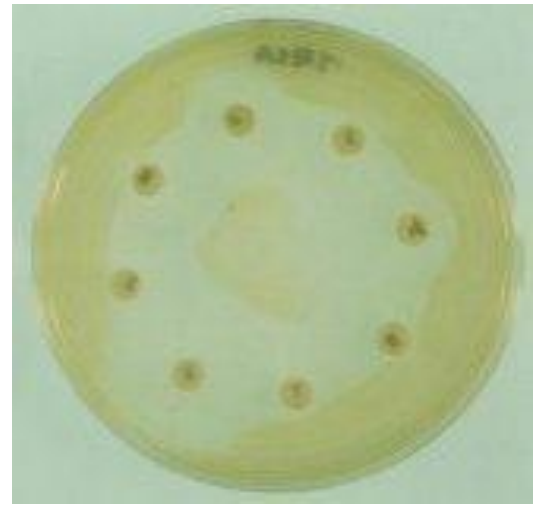

Figure 1. Photograph of petri dish showing disk diffusion method

\section{Minimum inhibitory concentrations (MIC) and minimum bactericidal concentrations (MBC)}

Minimum inhibitory concentrations (MICs) are known as the least concentration of an antimicrobial substance that will inhibit the visible growth of a microorganism after overnight incubation. Minimum bactericidal concentrations (MBCs) is the lowest concentration of an antimicrobial that will prevent the growth of an organism after subculture on to antibiotic-free media (Andrews 2001).

The active extracts were tested for MICs and MBCs. The MICs were set using a two-fold serial dilution method in a peptone water solution for bacterial of the active extracts to give a final extract with a concentration of between 1.74 and $8000 \mu \mathrm{g} / \mathrm{mL}$. Each tube was inoculated with $0.1 \mathrm{~mL}$ of standardized bacterial suspension $\left(1 \times 10^{8}\right.$ $\mathrm{CFU} / \mathrm{mL}$ ). The cultures were incubated for $24 \mathrm{hrs}$ at $37^{\circ} \mathrm{C}$ for bacteria. The first tube displaying no growth was taken as the MICs. MBCs were determined by sub-culturing all the tubes $(0.1 \mathrm{~mL})$ showing no growth on Nutrient Agar (NA) for bacteria. The first plate showing no growth was considered as the $\mathrm{MBC}$ after $48 \mathrm{hrs}$ at $37^{\circ} \mathrm{C}$ (Michael et al. 2003).

\section{Disc diffusion and MIC ratings of the extracts}

The negative controls showed no inhibition as opposed to the positive control using the standard antibiotic discs (Oxoid). Three replicates represent the mean zone of inhibition. A clearing zone of $9 \mathrm{~mm}$ or higher for either Gram-positive and gram-negative bacteria was used as the criterion for designating significant antibacterial (Faizi et al. 2003). The extracts that showed MIC lower than 100 $\mu \mathrm{g} / \mathrm{mL}$ were considered as having very high antimicrobial activity (AA); from $100-500 \mu \mathrm{g} / \mathrm{mL}$ has high AA; 500$1000 \mu \mathrm{g} / \mathrm{mL}$ has moderate; $1000-4000 \mu \mathrm{g} / \mathrm{mL}$, has low AA and anything above this range, the extracts were considered inactive for bacteria.

\section{Isolation of compounds from Ochna thomasiana}

The extracts of DCM and EtOAc from root bark were fractioned by $\mathrm{CC}$ of $4.75 \mathrm{~cm}$, and $5.75 \mathrm{~cm}$ in diameter, respectively with each height measured $70 \mathrm{~cm}$. The smaller $\mathrm{CC}$ of $2.75 \mathrm{~cm}$ in diameter and a height of $40 \mathrm{~cm}$ on silica gel 60 mesh $0.6 \mathrm{~nm}$ (230-400) was used for further purification, and the samples were eluted with varying

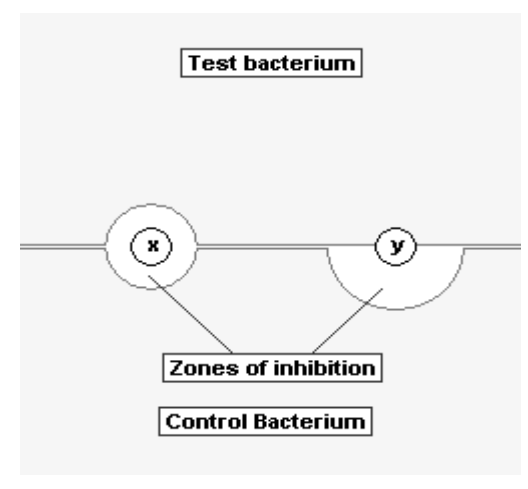

Figure 2. Test of the bacterium

concentrations of hexane, DCM, EtOAc, and $\mathrm{MeOH}$ mixtures until $100 \%$ of each solvent was achieved.

Packing of both types of columns was made using the slurry method with silica gel suspended in the less polar solvent in the solvent system. The mass of the silica gel packed measured in the ratio of 30:1 to the mass of the extract to be loaded. The extract was dissolved in the minimum possible solvent that dissolved it, then mixed with an equal amount of the silica gel used in the CC, ground into fine powder form and dry up by evaporating in a vacuum evaporator to remove the excess solvent. The powder was loaded at the top of the column, covered by a small amount of dry silica gel and covered with a piece of cotton wool to reduce the disturbance of the sample when the eluting solvent is added. The eluting solvent was allowed to trickle down dropwise from a separating funnel to the column to avoid pouring of the solvents directly into the column.

Analytical TLC $60 \mathrm{~F}_{254}$ plates were used throughout the purification process. These were mainly for the establishment of optimum solvent systems for the separations and purification of isolated compounds. Spots on the chromatograms were visualized under UV light at $\lambda$ 254 and $365 \mathrm{~nm}$ for UV active compounds and visualized upon development by spraying $p$-anisaldehyde separately and heating for about $10 \mathrm{~min}$ at $110{ }^{\circ} \mathrm{C}$ in an oven. Fractions that had same $\mathrm{Rf}$ were combined and concentrated at the same time to give pure compounds or semi-pure compounds for further purification. Further purifications steps included centrifuging, recrystallization, Sephadex column, and PTLC. The Sephadex (LH-20) columns were run using 1:1 (DCM: $\mathrm{MeOH})$.

DCM extract from the root $(9.65 \mathrm{~g})$ of the $O$. thomasiana proceed to fractionation by column chromatography on silica gel with a Hex:DCM-DCM: $\mathrm{MeOH}$ gradient (100:0-0:100) to yield $209 \times 25 \mathrm{~mL}$ fractions. Three fractions were acquired based on $R_{f}$ portrayed on the TLC profiles. Fractions 1-167 were pooled together since they gave a purple color on spraying with $p$-anisaldehyde followed by heating in the oven for 10 minutes at $110^{\circ} \mathrm{C}$. These fractions were generated at different solvent mixtures of hexane and DCM that range from 4:1 (Hex: DCM) to 100\% DCM. Fractions 168-181 were pooled at the same time and was obtained at 19:1 
(DCM: $\mathrm{MeOH}$ ). These fractions had purple and red-brown pigmentation after spraying with spraying reagent.

Fractions 182-209 were pooled together to form the third fraction which had yellow and red-brown pigmentation after spraying the TLC plate with $p$ anisaldehyde followed by heating in the oven for 5-10 minutes. This fraction was obtained at 9:1 of DCM: $\mathrm{MeOH}$. The first fraction (5-167) was rinsed with $\mathrm{MeOH}$ to remove brown coloration obtained from the column followed by hexane to make the crystals clean. Three other fractions were collected, but based on the TLC profile they all had two spots each. The white crystals obtained were dissolved in chloroform and then recrystallized. Decanting was done leaving behind the sharp white crystals of the compound of $\mathrm{MJ} / \mathrm{RD} / \mathrm{OTO}_{1}$ (75) of $3 \mathrm{~g}$. PTLC was done on one of the resulting three fractions to isolate $14.5 \mathrm{mg}$ of $\mathrm{MJ} / \mathrm{RD} / \mathrm{OT}_{10}$ (a mixture of 23 and 74 ) at $100 \%$ DCM. The other two portions were separated using Sephadex (L-20) column 1:1 (DCM: MeOH) and fractions 30 and 40were obtained. A PTLC was done on these combined fractions from the Sephadex column using 10\% MeOH/DCM, resulting in a white cream compound $\mathrm{MJ} / \mathrm{RD} / \mathrm{OTO}_{2}$ (17).

Ethyl acetate extract $(18.6 \mathrm{~g})$ of the root bark was proceeded to regular CC on silica gel with a DCM:EtOAc: $\mathrm{MeOH}$ gradient (100:0-0:100) to obtain 43 x $200 \mathrm{~mL}$ fractions. The fractions 1-5 obtained at 43:7 (DCM: EtOAc), 6-9 obtained at 5:3 (DCM: EtOAc), ten obtained at 11:9 (DCM: EtOAc), 11-15 obtained at 23:27 (DCM: EtOAc) and 16-43 were not incorporated because tailing produced in the TLC profiles. Pigmentation created after spraying with the $p$-anisaldehyde was dark brown. The fraction $11-15$ of $9.36 \mathrm{~g}$ was proceeded to a smaller $\mathrm{CC}$ to yield four other smaller fractions. The fractions 1-4 isolated at 19:1 (DCM: EtOAc), portion 5-11 obtained at 7:3 (DCM: EtOAc), portion 12-15 obtained at 5:3 (DCM: EtOAc), was separated further using Sephadex (L-20) column 1:1 (DCM: $\mathrm{MeOH}$ ) to receive 21 fractions which were of the same Rf. A PTLC was developed for the combined portion from the Sephadex using 9:1 (DCM: $\mathrm{MeOH})$, and $15.3 \mathrm{mg}$ of compound $18\left(\mathrm{MJ} / \mathrm{RE} / \mathrm{OTO}_{6}\right)$ was obtained. The same procedure above was used for fractions 6-9 to acquired $13.2 \mathrm{mg}$ of compound $20\left(\mathrm{MJ} / \mathrm{RE} / \mathrm{OTO}_{5}\right)$ and $14.5 \mathrm{mg}$ compound $17\left(\mathrm{MJ} / \mathrm{RE} / \mathrm{OTO}_{2}\right)$. Compound 17 was obtained from both DCM and EtOAc root bark extracts.

\section{Physical and spectroscopic data of isolated compounds Compound 18; Lophirone A}

Cream white crystals $(\mathrm{MeOH}), \mathrm{Mp} 211-213^{\circ} \mathrm{C}$; UV $(\mathrm{MeOH}) \lambda_{\max } 237,335 \mathrm{~nm} ;{ }^{1} \mathrm{H}$ NMR $(600 \mathrm{MHz}, \mathrm{MeOD}): \delta$ $8.25\left(1 \mathrm{H}, s, \beta_{1}\right), 6.68\left(1 \mathrm{H}, d, J=2.2, \mathrm{~B}_{1} \mathrm{H}_{3}\right), 7.87(1 \mathrm{H}, d$, $J=8.9 \mathrm{~Hz}, \mathrm{~B} 1 \mathrm{H} 6), 6.83(1 \mathrm{H}, d \mathrm{~d}, J=2.2,8.9, \mathrm{~B} 1 \mathrm{H} 5), 5.97$ $\left(1 \mathrm{H}, d, J=12.16, \mathrm{H}-\alpha_{1}\right), 4.67\left(1 \mathrm{H}, d, J=12.16, \mathrm{H}-\beta_{1}\right), 6.59$ (4H, d, J=8.4, H-C3, A1C5, A2,7.16 (4H, d, J=8.4, H-C2, Al C6, A2), 6.33 (1H, dd, J=2.3, 9.0, B2H5),6.12 (1H, d, $J=2.3, \mathrm{~B} 2 \mathrm{H} 3), 8.14\left(1 \mathrm{H}, d, J=9.0, \mathrm{~B}_{2} \mathrm{H}_{6}\right) .{ }^{13} \mathrm{C}$ NMR $(150$ $\mathrm{MHz}, \mathrm{MeOD}): \delta 157.4\left(\beta_{1}\right), 122.6\left(\alpha_{1}\right), 177.1\left(\mathrm{~s}, \mathrm{C}_{1}\right)$, $117.1\left(\mathrm{~s}, \mathrm{~B}_{1} \mathrm{C}_{1}\right), 103.5\left(d, \mathrm{~B}_{2} \mathrm{C}_{3}\right), 164.7\left(\mathrm{~B}_{1} \mathrm{C}_{4}\right), 159.4(\mathrm{~s}$, B1C6), $116.5\left(d, \mathrm{~A}_{1} \mathrm{C}_{5}\right), 128.2\left(d, \mathrm{~A}_{1} \mathrm{C}_{2}\right), 54.3\left(d, \beta_{1}\right), 44.7$

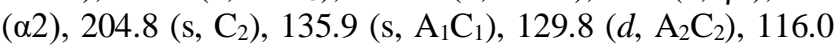

$\left(d, \mathrm{~A}_{2} \mathrm{C}_{3}\right), 156.7\left(\mathrm{~s}, \mathrm{~A}_{1} \mathrm{C}_{4}\right), 134.9\left(\mathrm{~s}, \mathrm{~A}_{2} \mathrm{C}_{1}\right), 130.5(d$, $\left.\mathrm{A}_{2} \mathrm{C}_{6}\right), 116.1\left(d, \mathrm{~A}_{2} \mathrm{C}_{5}\right), 114.3$ (s, B2C1),159.4 (B1C6), $166.9\left(s, \mathrm{~B}_{2} \mathrm{C}_{2}\right), 103.5\left(d, \mathrm{~B}_{2} \mathrm{C}_{3}\right), 166.8\left(\mathrm{~s}, \mathrm{~B}_{2} \mathrm{C}_{4}\right), 109.2(d$, $\left.\mathrm{B}_{2} \mathrm{C}_{5}\right), 134.4\left(d, \mathrm{~A}_{2} \mathrm{C}_{1}\right)$; IR (KBr) nmax 3751, 3368, 2728, $1626,1510,1454,1364,1238,831 \mathrm{~cm}^{-1}$ (Tables 1-10).

\section{Compound 20; Afzelone D dimethylether}

Yellow crystals $(\mathrm{MeOH}), \mathrm{Mp} 172-175^{\circ} \mathrm{C}$; Formula: $\mathrm{C}_{33} \mathrm{H}_{28} \mathrm{O}_{8},{ }^{1} \mathrm{H}$ NMR (400 MHz, MeOD): $\delta 8.22(1 \mathrm{H}, \mathrm{s}$, $\left.\beta_{1}\right), 6.69\left(1 \mathrm{H}, d, J=2.2, \mathrm{~B}_{1} \mathrm{H}_{3}\right), 7.85(1 \mathrm{H}, \mathrm{d}, \mathrm{J}=8.9 \mathrm{~Hz}$, B1H6), $6.83\left(1 \mathrm{H}, d d, J=2.2,8.9, \mathrm{~B}_{1} \mathrm{H}_{5}\right), 7.10(4 \mathrm{H}, d, J=8.4$, $\mathrm{H}-\mathrm{C} 2, \mathrm{~A} 1 \mathrm{C} 6, \mathrm{~A} 2), 6.02\left(1 \mathrm{H}, d, J=12.2, \mathrm{H}-\alpha_{1}\right), 4.70(1 \mathrm{H}$, $\left.d, J=12.2, \mathrm{H}-\beta_{1}\right), 6.56\left(4 \mathrm{H}, d, J=8.4, \mathrm{H}-\mathrm{C}_{3}, \mathrm{~A}_{1} \mathrm{C}_{5}, \mathrm{~A}_{2}\right)$, $6.44(1 \mathrm{H}, d \mathrm{~d}, J=2.3,9.0, \mathrm{~B} 2 \mathrm{H} 5), 6.28(1 \mathrm{H}, d, J=2.3$, $\mathrm{B} 2 \mathrm{H} 3), 8.14\left(1 \mathrm{H}, d, J=9.0, \mathrm{~B}_{2} \mathrm{H}_{6}\right), 3.76\left(3 \mathrm{H}, \mathrm{A}_{1} \mathrm{C}_{4}, \mathrm{OMe}\right)$, $3.75\left(3 \mathrm{H}, \mathrm{A}_{2} \mathrm{C}_{4}, \mathrm{OMe}\right), 3.59\left(3 \mathrm{H}, \mathrm{OMe}_{2} \mathrm{C}_{4}\right) \cdot{ }^{13} \mathrm{C} \mathrm{NMR}$ (100 MHz, MeOD): $\delta 157.4\left(\beta_{1}\right), 177.1\left(s, \mathrm{C}_{1}\right), 117.1(s$, $\left.\mathrm{B}_{1} \mathrm{C}-1\right), 122.6(\alpha 1), 159.4\left(s, \mathrm{~B}_{1} \mathrm{C}_{6}\right), 103.5\left(d, \mathrm{~B}_{2} \mathrm{C}_{3}\right), 164.7$ $\left(\mathrm{B}_{1} \mathrm{C}_{4}\right), 116.5\left(d, \mathrm{~A}_{1} \mathrm{C}_{5}\right), 135.9(\mathrm{~s}, \mathrm{~A} 1 \mathrm{C} 1), 128.2\left(d, \mathrm{~A}_{1} \mathrm{C}_{2}\right)$, $44.7\left(\alpha_{2}\right), 54.3\left(d, \mathrm{C}_{\beta 1}\right), 204.8\left(s, \mathrm{C}_{2}\right), 129.8\left(\mathrm{~d}, \mathrm{~A}_{2} \mathrm{C}_{2}\right)$, $116.0\left(d, \mathrm{~A}_{2} \mathrm{C}_{3}\right), 156.7\left(\mathrm{~s}, \mathrm{~A}_{1} \mathrm{C}_{4}\right), 130.5$ (d, A2C6), 134.9 (s, $\mathrm{A} 2 \mathrm{C} 1), \quad 116.1 \quad\left(d, \quad \mathrm{~A}_{2} \mathrm{C}_{5}\right), \quad 159.4 \quad\left(\mathrm{~B}_{1} \mathrm{C}_{6}\right), 114.3 \quad(s$, $\left.\mathrm{B}_{2} \mathrm{C}_{1}\right), 166.8(\mathrm{~s}, \mathrm{~B} 2 \mathrm{C} 4), 166.9\left(s, \mathrm{~B}_{2} \mathrm{C}_{2}\right), 103.5\left(d, \mathrm{~B}_{2} \mathrm{C}_{3}\right)$, $109.2\left(d, \mathrm{~B}_{2} \mathrm{C}_{5}\right), 134.4\left(d, \mathrm{~A}_{2} \mathrm{C}_{1}\right), 56.0\left(q, \mathrm{~A}_{2} \mathrm{C}_{4} \mathrm{OMe}\right), 56.1$ ( $\left.q, \mathrm{~A}_{1} \mathrm{C}_{4} \mathrm{OMe}\right), 55.5$ ( $\left.q, \mathrm{~B}_{2} \mathrm{C}_{4} \mathrm{OMe}\right)$; IR (KBr) nmax 3855, $3443,2933,1611,1510,1452,1365,1256,1026,831 \mathrm{~cm}^{-1}$ (Table 6)

\section{Compound 17; Calodenone}

Cream white crystals $(\mathrm{MeOH}), \mathrm{Mp} 172-175^{\circ} \mathrm{C}$; Formula: $\mathrm{C}_{31} \mathrm{H}_{24} \mathrm{O}_{8},{ }^{1} \mathrm{H}$ NMR (400 MHz, MeOD): $\delta 8.22$ $\left(1 \mathrm{H}, s, \beta_{1}\right), 6.83\left(1 \mathrm{H}, d, J=2.2, \mathrm{~B}_{1} \mathrm{H}_{3}\right), 7.87(1 \mathrm{H}, \mathrm{d}$, $\mathrm{J}=8.9 \mathrm{~Hz}, \mathrm{~B} 1 \mathrm{H} 2), 6.72\left(1 \mathrm{H}, d d, J=2.2,8.9, \mathrm{~B}_{1} \mathrm{H}_{5}\right), 6.03(1 \mathrm{H}$, $\left.d, J=12.2, \mathrm{H}-\alpha_{2}\right), 4.67\left(1 \mathrm{H}, d, J=12.2, \mathrm{H}-\beta_{2}\right), 7.15(4 \mathrm{H}, d$, $\left.J=8.4, \mathrm{H}-\mathrm{C}_{2}, \mathrm{~A}_{1} \mathrm{C}_{6}, \mathrm{~A}_{2}\right), 6.55\left(4 \mathrm{H}, d, J=8.4, \mathrm{H}-\mathrm{C}_{3}, \mathrm{~A}_{1} \mathrm{C}_{5}\right.$, $\left.\mathrm{A}_{2}\right), 6.60(1 \mathrm{H}, d d, J=2.3,9.0, \mathrm{~A} 1 \mathrm{H} 5), 6.29(1 \mathrm{H}, d, J=2.3$, $\mathrm{B} 2 \mathrm{H} 3), 8.21\left(1 \mathrm{H}, d, J=9.0, \mathrm{~B}_{2} \mathrm{H}_{6}\right), 3.76\left(3 \mathrm{H}, \mathrm{OMe} \mathrm{A}_{2} \mathrm{C}_{4}\right)$. ${ }^{13} \mathrm{C}$ NMR (100 MHz, MeOD): $\delta 157.4\left(\beta_{1}\right), 122.6\left(\alpha_{1}\right)$, $177.1\left(\mathrm{~s}, \mathrm{C}_{1}\right), 117.1 \quad\left(\mathrm{~s}, \mathrm{~B}_{1} \mathrm{C}_{1}\right), 164.7$ (B1C4),159.4 (s, $\left.\mathrm{B}_{1} \mathrm{C}_{6}\right), 103.5\left(\mathrm{~d}, \mathrm{~B}_{2} \mathrm{C}_{3}\right), 116.5\left(d, \mathrm{~A}_{1} \mathrm{C}_{5}\right), 128.2\left(d, \mathrm{~A}_{1} \mathrm{C}_{2}\right)$, $44.7\left(\alpha_{2}\right), \quad 54.3 \quad\left(\mathrm{~d}, \quad \mathrm{C}_{\beta 1}\right), \quad 204.8 \quad\left(\mathrm{~s}, \quad \mathrm{C}_{2}\right), 156.7 \quad(\mathrm{~s}$, $\mathrm{A} 1 \mathrm{C} 4), 135.9\left(\mathrm{~s}, \mathrm{~A}_{1} \mathrm{C}_{1}\right), 129.8\left(d, \mathrm{~A}_{2} \mathrm{C}_{2}\right), 116.0\left(d, \mathrm{~A}_{2} \mathrm{C}_{3}\right)$, $134.9\left(\mathrm{~s}, \mathrm{~A}_{2} \mathrm{C}_{1}\right), 130.5\left(d, \mathrm{~A}_{2} \mathrm{C}_{6}\right), 159.4$ (B1C6), 116.1 (d, $\left.\mathrm{A}_{2} \mathrm{C}_{5}\right), 114.3\left(s, \mathrm{~B}_{2} \mathrm{C}_{1}\right), 166.9\left(s, \mathrm{~B}_{2} \mathrm{C}_{2}\right), 103.5\left(d, \mathrm{~B}_{2} \mathrm{C}_{3}\right)$, $166.8\left(\mathrm{~s}, \mathrm{~B}_{2} \mathrm{C}_{4}\right), 134.4$ (d, A2C1),109.2 (d, B2C5), 56.1 (q, $\mathrm{A}_{1} \mathrm{C}_{4} \mathrm{OMe}$ ); IR (KBr) nmax 3753, 3423, 2747, 1626, 1510, $1454,1369,1253,1024,749,829 \mathrm{~cm}^{-1}$ (Table 7).

\section{Compound 23; $\beta$-sitosterol}

White amorphous solid $\left(\mathrm{CHCl}_{3}\right) \mathrm{Mp} 130-134^{\circ} \mathrm{C}$; Mol. Wts: 415 and Mol. Formula: $\mathrm{C}_{29} \mathrm{H}_{50} \mathrm{O}\left(\mathrm{CHCl}_{3}\right)$. IR $(\mathrm{KBr})$ $v_{\max }: 3400 \mathrm{~cm}^{-1}$ (OH- stretching), $2899 \mathrm{~cm}^{-1}(\mathrm{CH}-$ stretching), $1679 \mathrm{~cm}^{-1}, 1460 \mathrm{~cm}^{-1} \mathrm{C}=\mathrm{C}$ stretching, $1041 \mathrm{~cm}^{-}$ ${ }^{1} \mathrm{C}-\mathrm{O}-\mathrm{C}$ stretching. Mass spectra: m/z: $415\left(\mathrm{M}+, \mathrm{C}_{20} \mathrm{H}_{50} \mathrm{O}\right)$, $399\left(\mathrm{M}+-\mathrm{CH}_{3}\right), 381(\mathrm{M}+\mathrm{CH}, \mathrm{OH}), 376(\mathrm{M}+\mathrm{H} 2 \mathrm{O}), 320$, 303, 273, 255. ${ }^{1} \mathrm{H} \mathrm{NMR:}\left(\mathrm{CDCl}_{3}, 400 \mathrm{MHz}\right) 0.68 \mathrm{ppm}$ $\left(3 \mathrm{H}, s, \mathrm{C}_{18} \mathrm{H}\right), 0.85\left(\mathrm{H}_{26}\right), 0.81(\mathrm{H} 29), 0.80(\mathrm{H} 27), 1.01\left(\mathrm{H}_{19}\right)$, $1.02\left(3 \mathrm{H}, s, \mathrm{C}_{21} \mathrm{H}\right), 3.53\left(1 \mathrm{H}, m, \mathrm{C}_{3} \mathrm{H}\right), 5.36(1 \mathrm{H}, t, J=6$ $\left.\mathrm{Hz}, \mathrm{C}_{6} \mathrm{H}\right)$. Other peaks are observed at $\delta 0.80-\delta 2.4 .{ }^{13} \mathrm{C}$ NMR $\left(\mathrm{CDCl}_{3}, 100 \mathrm{MHz}\right)$ of 23: ${ }^{13} \mathrm{C}$ NMR has given signal 
at $140.8\left(\mathrm{C}_{5}\right), 121.7\left(\mathrm{C}_{6}\right), 33.7\left(\mathrm{C}_{22}\right), 26.1\left(\mathrm{C}_{23}\right), 56.9$ $\left(\mathrm{C}_{14}\right), 56.1(\mathrm{C} 17), 71.8\left(\mathrm{C}_{3}\right), 42.3\left(\mathrm{C}_{14}\right), 50.2\left(\mathrm{C}_{9}\right), 36.2$ $\left(\mathrm{C}_{20}\right), 39.8\left(\mathrm{C}_{12}\right), 42.3\left(\mathrm{C}_{13}\right), 42.3\left(\mathrm{C}_{4}\right), 37.2\left(\mathrm{C}_{1}\right), 30.5$ $\left(\mathrm{C}_{10}\right), 31.9\left(\mathrm{C}_{8}\right), 33.7 \quad(\mathrm{C} 22), 36.2(\mathrm{C} 20), 31.9$ (C8),31.7 (C7), $29.2\left(\mathrm{C}_{25}\right), 28.2\left(\mathrm{C}_{16}\right), 31.7\left(\mathrm{C}_{2}\right), 24.3\left(\mathrm{C}_{15}\right), 23.1$ $\left(\mathrm{C}_{28}\right)$, $21.1\left(\mathrm{C}_{11}\right), 21.2,19.4(\mathrm{C} 19), 19.0(\mathrm{C} 27), 18.8\left(\mathrm{C}_{21}\right)$, 11.9, $12.0\left(\mathrm{C}_{18}\right), 12.0,11.7\left(\mathrm{C}_{29}\right)$ (Table 8).

\section{Compound 74; Stigmasterol}

White amorphous solid $\left(\mathrm{CHCl}_{3}\right) \mathrm{Mp} 130-134^{\circ} \mathrm{C}$; Mol. Wts: 413 and Mol. Formula: $\mathrm{C}_{29} \mathrm{H}_{48} \mathrm{O}\left(\mathrm{CHCl}_{3}\right)$. IR $(\mathrm{KBr})$ $v_{\max }: 3400 \mathrm{~cm}^{-1}$ (OH- stretching), $2899 \mathrm{~cm}^{-1}(\mathrm{CH}-$ stretching), $1679 \mathrm{~cm}^{-1}, 1460 \mathrm{~cm}^{-1} \mathrm{C}=\mathrm{C}$ stretching, $1041 \mathrm{~cm}^{-}$ ${ }^{1} \mathrm{C}-\mathrm{O}-\mathrm{C}$ stretching. Mass spectra: $\mathrm{m} / \mathrm{z}: 414\left(\mathrm{M}+, \mathrm{C}_{20} \mathrm{H}_{50} \mathrm{O}\right)$, $399\left(\mathrm{M}+-\mathrm{CH}_{3}\right), 376\left(\mathrm{M}+\mathrm{H}_{2} \mathrm{O}\right), 381(\mathrm{M}+\mathrm{CH}, \mathrm{OH}), 320$ 303, 273, 255. ${ }^{1} \mathrm{H}$ NMR: $\left(\mathrm{CDCl}_{3}, 400 \mathrm{MHz}\right) 0.68 \mathrm{ppm}$ $\left(3 \mathrm{H}, s, \mathrm{C}_{18} \mathrm{H}\right), 0.85\left(\mathrm{H}_{26}\right), 0.81(\mathrm{H} 29), 0.80(\mathrm{H} 27), .01\left(\mathrm{H}_{19}\right)$ $3.53\left(1 \mathrm{H}, m, \mathrm{C}_{3} \mathrm{H}\right), 5.36\left(1 \mathrm{H}, t, J=6 \mathrm{~Hz}, \mathrm{C}_{6} \mathrm{H}\right), 1.02(3 \mathrm{H}$, $\mathrm{s}, \mathrm{C} 21 \mathrm{H}), 02\left(1 \mathrm{H}, m, \mathrm{H}_{23}\right), 5.15\left(1 \mathrm{H}, m, \mathrm{H}_{22}\right)$. Other peaks appeared at $\delta 0.80-\delta 2.4 .{ }^{13} \mathrm{C} \mathrm{NMR}\left(\mathrm{CDCl}_{3}, 100 \mathrm{MHz}\right)$ of 74: ${ }^{13} \mathrm{C}$ NMR has given signal at 140.8 $\left(\mathrm{C}_{5}\right), 138.3(\mathrm{C} 22), 121.7\left(\mathrm{C}_{6}\right), 129.3\left(\mathrm{C}_{23}\right), 56.8\left(\mathrm{C}_{14}\right) 71.8\left(\mathrm{C}_{3}\right)$, $42.3\left(\mathrm{C}_{14}\right), 56.1\left(\mathrm{C}_{17}\right), 50.2\left(\mathrm{C}_{9}\right), 40.5\left(\mathrm{C}_{20}\right), 39.7\left(\mathrm{C}_{12}\right)$, $42.3\left(\mathrm{C}_{13}\right), 42.3\left(\mathrm{C}_{4}\right), 37.2\left(\mathrm{C}_{1}\right), 31.9(\mathrm{C} 8), 30.5(\mathrm{C} 10), 40.5$ $\left(\mathrm{C}_{20}\right), 31.7\left(\mathrm{C}_{7}\right), 31.9\left(\mathrm{C}_{8}\right), 31.9\left(\mathrm{C}_{25}\right), 28.7\left(\mathrm{C}_{16}\right), 31.7\left(\mathrm{C}_{2}\right)$, $24.3\left(\mathrm{C}_{15}\right), 25.4\left(\mathrm{C}_{28}\right), 21.2(\mathrm{C} 27), 21.1\left(\mathrm{C}_{11}\right), 19.4\left(\mathrm{C}_{19}\right)$, $21.1\left(\mathrm{C}_{21}\right), 11.9\left(\mathrm{C}_{18}\right), 12.0\left(\mathrm{C}_{29}\right)$ (Table 8).

\section{Compound 75; $3 \beta$-acetyl-24-ethylfriedelane}

White amorphous solid $\left(\mathrm{CHCl}_{3}\right), \mathrm{Mp} 266-267^{\circ} \mathrm{C}$; IR $\left((\mathrm{KBr}) v_{\max }: 3619,3471,2725,2869,1448,1384,1360\right.$, $1172,1089,1020,1000,979$, and $720 ;{ }^{1} \mathrm{H}-\mathrm{NMR}(600$ $\left.\mathrm{MHz} ; \mathrm{CDCl}_{3} ; \mathrm{ppm}\right) \delta 3.70(1 \mathrm{~s} ; \mathrm{H}-3 ; 1 \mathrm{H})$ and $0.83-0.97$ (superposed signals) ${ }^{1} \mathrm{H}$ NMR $\left(\mathrm{CDCl}_{3}, 600 \mathrm{MHz}\right), 0.82$ $\left(3 \mathrm{H}, s, \mathrm{CH}_{3}-25\right), 0.86\left(3 \mathrm{H}, s, \mathrm{CH}_{3}-24\right), 0.93\left(3 \mathrm{H}, s, \mathrm{CH}_{3}-\right.$ 28), $1.03\left(6 \mathrm{H}, s, \mathrm{CH}_{3}-27, \mathrm{CH} 3-28\right), 0.97\left(3 \mathrm{H}, \mathrm{s}, \mathrm{CH}_{3}-32\right)$, $1.00\left(3 \mathrm{H}, s, \mathrm{CH}_{3}-23\right), 1.23\left(3 \mathrm{H}, s, \mathrm{CH}_{3}-30\right), 2.00(3 \mathrm{H}, s$, $\left.\mathrm{CH}_{3}\right), 4.10,\left(1 \mathrm{H}, d, 14.0, \mathrm{H}_{3}\right) .{ }^{13} \mathrm{C}-\mathrm{NMR}\left(150 \mathrm{MHz} ; \mathrm{CDCl}_{3}\right.$; ppm) $\delta 72.8\left(\mathrm{C}_{3}\right), 61.3\left(\mathrm{C}_{10}\right), 53.2\left(\mathrm{C}_{8}\right), 37.1\left(\mathrm{C}_{4}\right), 60.4$ (C5),42.8 ( $\left.\mathrm{C}_{18}\right), 41.7\left(\mathrm{C}_{6}\right), 39.7\left(\mathrm{C}_{14}\right), 39.3\left(\mathrm{C}_{22}\right), 38.4$ $\left(\mathrm{C}_{13}\right), 37.1\left(\mathrm{C}_{32}\right), 37.8\left(\mathrm{C}_{9}\right), 35.6\left(\mathrm{C}_{16}\right), 35.3\left(\mathrm{C}_{11}\right), 35.2$ (C2), $35.2\left(\mathrm{C}_{19}\right), 35.0\left(\mathrm{C}_{30}\right), 32.8\left(\mathrm{C}_{21}\right), 32.3\left(\mathrm{C}_{15}\right), 32.1$ $\left(\mathrm{C}_{28}\right), 31.8\left(\mathrm{C}_{29}\right), 30.6\left(\mathrm{C}_{12}\right), 30.0\left(\mathrm{C}_{17}\right), 28.2\left(\mathrm{C}_{20}\right), 20.1$ $\left(\mathrm{C}_{26}\right), 18.2(\mathrm{C} 25), 18.6(\mathrm{C} 27), 17.5\left(\mathrm{C}_{7}\right), 16.4\left(\mathrm{C}_{24}\right), 14.2$ $(31), 15.8\left(C_{1}\right)$, and $11.6\left(C_{23}\right)$ (Table 9$)$.

\section{RESULTS AND DISCUSSION}

\section{Plant material yield of extracts}

The $\mathrm{MeOH}$ extracts of root and stem recorded the highest return (Table 1). The root bark of $O$. thomasiana was more abundant in metabolites that were soluble in the $\mathrm{MeOH}$ solvents used in the extraction compared DCM and EtOAc solvents. It also showed that the yield increased with increased polarity, as in the case of the root bark extract, which increased from $1.15 \%$ yield with DCM as a solvent to $27.0 \%$ with $\mathrm{MeOH}$ as the solvent. This suggests that both the stem and root bark of $\mathrm{MeOH}$ extracts contain more polar compounds. Before embarking on fractionation of the crude extracts, these extracts were subjected to various antibacterial tests. The total yield of the extract from the stem bark was $22.45 \%$ while that of the root bark was $34.6 \%$.

\section{Antibacterial disc diffusion screening test for the $\boldsymbol{O}$. thomasiana extracts \\ The DCM extracts}

The stem DCM extracts did not show any activity against $P$. aeruginosa, S. typhi, and S.aureus. The root DCM extracts showed slight activity against $B$. subtilis, but no activity on $P$. aeruginosa, S. typhi, and $S$. aureus (Table 2).

\section{The ethyl acetate extracts}

The root and the stem bark extracts of $O$. thomasiana exhibited moderate activity against $S$. aureus and B. subtilis (Table 2). There was no antibacterial activity observed for the acetate extracts of the plant against $S$. typhi and $P$. aeruginosa.

\section{The methanol extracts}

There was high activity against $B$. subtilis and $S$. aureus for the root bark extracts of $O$. thomasiana (Table 2). The root and stem methanol extracts for the plant were highly active against $B$. subtilis. Methanol extracts demonstrated broad spectra of activity against the tested organisms, except for $P$. aeruginosa and S.typhi. The $\mathrm{MeOH}$ root extract of the plant had moderate activity at the concentrations tested with a mean zone of inhibition diameter greater than or equal to $15 \mathrm{~mm}$.

The selective effects of the extracts $S$. aureus and $B$. subtilis suggest that the extracts may serve as a source of compounds that can be used to combat infection caused by these organisms (Tania et al. 2000).

Table 1. Plant material yield of Ochna thomasiana extracts

\begin{tabular}{llll}
\hline Plant part & Extract & Mass of extract $(\mathrm{g})$ & \% yield \\
\hline \multirow{3}{*}{ Stem bark } & DCM & 71 & 1.10 \\
& EtOAc & 100 & 1.65 \\
& MeOH & 741 & 19.7 \\
Root bark & DCM & 46 & 1.15 \\
& EtOAc & 167 & 6.65 \\
& $\mathrm{MeOH}$ & 631 & 27.0 \\
\hline
\end{tabular}

Table 2. The inhibition zones (in mm) of crude extracts of Ochna thomasiana

\begin{tabular}{lllll}
\hline Extract & S. aureus & B. subtilis & P. aeruginosa S. typhi \\
\hline EtOAc stem & 9 & 11 & 6 & 6 \\
MeOH stem & 10 & 14 & 6 & 6 \\
EtOAc root & 9 & 11 & 6 & 6 \\
MeOH root & 15 & 20 & 6 & 6 \\
$\begin{array}{l}\text { Control experiment } \\
\text { (+ve) }\end{array}$ & 20 (Tet) & 18 (Tet) & 19 (Tet) & 15 (Tet) \\
\hline Note: Bacillus & subtilis ATCC & 25726, Staphylococcus aureus \\
$\begin{array}{l}\text { ATCC 25724, } \\
\text { Salmonella typhi (clinical isolate), Tet: tetracycline }\end{array}$ &
\end{tabular}


Table 3. The MIC and MBC $(\mu \mathrm{g} / \mathrm{mL})$ of crude extracts of Ochna thomasiana

\begin{tabular}{llll}
\hline Extract & $\boldsymbol{\mu g} / \mathbf{m L}$ & S. aureus & B. subtilis \\
\hline EtOAc stem & MIC & 1000 & 1000 \\
& MBC & 8000 & 8000 \\
MeOH stem & MIC & 1000 & 1000 \\
& MBC & 8000 & 8000 \\
EtOAc root & MIC & 1000 & 1000 \\
& MBC & 8000 & 8000 \\
MeOH root & MIC & 500 & 500 \\
& MBC & 8000 & 8000 \\
\hline
\end{tabular}

\section{MIC and MBC of Ochna thomasiana}

The MIC and MBC results are also given in Table 3. $\mathrm{MeOH}$ root bark recorded the lowest MIC of $500 \mu \mathrm{g} / \mathrm{mL}$ against both B. subtilis and S. aureus. Both stem and root extract of EtOH and EtOAc respectively showed the highest MIC of $1000 \mu \mathrm{g} / \mathrm{mL}$ against $B$. subtilis. The activity against all the Gram-positive bacteria used was high in the stem and root extracts because all of them had MICs of 500-1000 $\mu \mathrm{g} / \mathrm{mL}$. The MBCs for all extracts were observed to be about $8000 \mu \mathrm{g} / \mathrm{mL}$; confirming that $O$. thomasiana had considerable antimicrobial activity against gram-positive tested microorganisms. The $\mathrm{MeOH}$ and EtOAc extract showed more activity against the tested organisms, as indicated by the susceptibility, MIC, and MBC tests. The result suggests that more of the bioactive chemical constituents present in the $\mathrm{MeOH}$ extracts. These could probably be polar or moderately-polar compounds such as flavonoids. All the EtOAc and $\mathrm{MeOH}$ extracts had no activity against all the Gram-negative bacteria tested.

\section{Structure elucidation}

The structure elucidation of compounds 17 to 75 employed IR spectroscopic, mass spectrometric spectral data, and interpretive NMR obtained for the purified compounds in comparison with literature values.

\section{Compound 18}

Compound 18 was isolated as a cream-white amorphous solid $(15.3 \mathrm{mg}$ ) from a $\mathrm{MeOH}$ soluble portion of EtOAc root bark extract (MeOH/DCM; 1:9) and with an $\mathrm{mp}$ of $211-213^{\circ} \mathrm{C}$. It had fluorescing characteristic under UV-light $(254 \mathrm{~nm})$ and produced a characteristic red colored spot when sprayed with $p$-anisaldehyde followed by heating at $110^{\circ} \mathrm{C}$ in the oven for $5-10$ minutes. The UV in $\mathrm{MeOH}$ displayed absorptions 237 and $335 \mathrm{~nm}$ suggestive of an isoflavonoid nucleus (Enas et al. 2012). The IR spectrum, comprised of absorption bands at $3252 \mathrm{~cm}^{-1}$ (C-H aromatic stretching), $831^{-1}$ (out of plane bend), $1625 \mathrm{~cm}^{-1}$ (aromatic $\mathrm{C}=\mathrm{C}$ ). The IR spectrum produced a broad $\mathrm{O}-\mathrm{H}$ absorption band at $3368 \mathrm{~cm}^{-1}$ and two carbonyl bands at $1625 \mathrm{~cm}^{-1}$.

The ${ }^{1} \mathrm{H},{ }^{1} \mathrm{H}$ COSY, and the ${ }^{1} \mathrm{H}$ NMR spectra displayed the presence of two $p$-substituted aromatic rings plus two trisubstituted phenyl ring systems. A singlet at $\delta 8.25$ is characteristic of $\mathrm{H}-\beta_{1}$ of a 3 -substituted benzopyran- 4-one moiety and the peaks at $\delta 6.01$ and 4.67 showed the presence of an AX system. The large coupling $(J=12.2 \mathrm{~Hz})$ seen between the latter two protons suggested that they are in an antiperiplanar (Table 4) (Mabry et al. 1970; Pegnyemb et al. 2003).

In the ${ }^{13} \mathrm{C}$ NMR spectrum, 22 peaks are corresponding to 26 carbon atoms with chemical shifts between $\delta 100$ to 170 indicating the presence of four aromatic ring systems in addition to the pyran-4-one ring (Markham et al. 1978). In the HSQC spectrum, the methine carbon atoms at $\delta 54.3$ and 44.7 were positively correlated to protons at $\delta 4.67$ and 6.01 , respectively. The two carbonyl carbon atoms resonated at $\delta 204.6$ and 177.1 and the downfield resonance of the later carbonyl carbon atom indicated that it is part of a 4-pyrone system.

The position of the two $p$-substituted aromatic ring systems ( $\mathrm{AA}^{\prime \prime} \mathrm{XX}$ ') is assigned by the long-range heteronuclear correlation [HMBC spectrum between $\mathrm{H}-\beta_{2}$ at $\delta 4.67$ and $\mathrm{A}_{2} \mathrm{C}_{1}$ and $\mathrm{A}_{1} \mathrm{C}_{1}$ at $\delta 134.9$ and 129.7 of ring $A_{1}$ and $A_{2}$, respectively. The result is indicative of the connection of both rings to $\mathrm{C}-\beta_{2}$; therefore, validating partial structure compound 18 (Figure 3). The downfield resonance of $\mathrm{B}_{1} \mathrm{H}_{6}$ at $\delta 7.89$ shows its proximity to the carbonyl and therefore, the second trisubstituted aromatic ring (appeared as ring $\mathrm{B}_{1}$ ) is not substituted at $\mathrm{C}_{5}$ (Abraham et al. 2003).

The presence of a singlet at around $\delta 8.25$ evidenced that $\mathrm{C}_{2}$ of the benzopyran moiety is not substituted. The long-range correlation between $\mathrm{H}-\alpha_{2}$ and $\mathrm{C}-\alpha_{1}$ confirms that $\mathrm{C}-\alpha_{2}$ linked to the benzopyran moiety through $\mathrm{C}-\alpha_{1}$. The long-term relationships between B1H6 confirmed the position of the first trisubstituted aromatic system and the carbonyl at $\delta 177.1$ suggesting that this particular ring $\mathrm{B}_{1}$ is part of a benzopyran moiety and therefore forms ring A of a flavonoid skeleton. Long-range correlations between $\mathrm{B}_{2} \mathrm{H}_{6}$ and a carbonyl at $\delta 204.7\left(\mathrm{C}_{2}\right)$ suggested the relationship of the second trisubstituted aromatic ring to this carbonyl, which in turn was also correlated to $\mathrm{H}-\mathrm{\alpha}_{2}$ as shown in partial structure and Table 5.

The assignment of compound 18 was with an agreement with the previously isolated from the stem bark of Lophira lanceolata (Ochnaceae) that has a molecular formula of C30H22O8 (Ghogomu et al. 1987). The NMR data of compound 18 were in agreement with those reported for the compound. The compound was confirmed to be a biflavonoid; Lophirone A and with the same structure as compound 18 based on the analysis of data above, and it is the first time the compound was isolated from this plant (Figure 5).

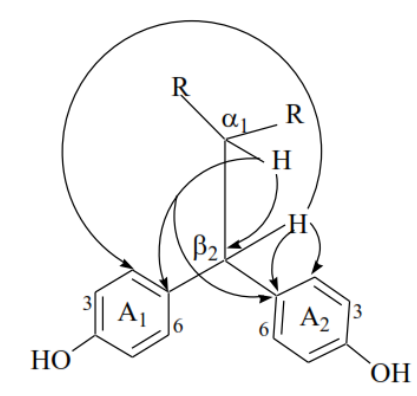

Figure 3. HMBC correlations in partial structure 18 
Table 4. ${ }^{1} \mathrm{H}$ NMR $\left(600 \mathrm{MHz}, \mathrm{CD}_{3} \mathrm{OD}\right)$ and $\mathrm{COSY}$ for compound 18

\begin{tabular}{llllll}
\hline Position & $\boldsymbol{\delta}$ Obs & Multiplicity & $\boldsymbol{J}_{\text {Obs }}(\mathbf{H z})$ & COSY & $\boldsymbol{\delta}$ Lit. \\
\hline$\beta_{1}$ & 8.25 & $s$ & & & 8.27 \\
$\mathrm{~B}_{1} 3$ & 6.85 & $d d$ & $8.9,2.2$ & & 6.72 \\
$\mathrm{~B}_{1} 5$ & 6.72 & $d$ & 2.2 & & 6.96 \\
$\mathrm{~B}_{1} 6$ & 7.89 & $d$ & 8.9 & $\mathrm{~B}_{2} \mathrm{H}_{3}$ & 7.74 \\
$\alpha_{2}$ & 6.01 & $d$ & 12.2 & $\alpha_{2}$ & 6.01 \\
$\mathrm{~B}_{2} 3$ & 6.14 & $d$ & 2.3 & & 6.19 \\
$\mathrm{~B}_{2} 5$ & 6.34 & $d d$ & $9.0,2.2$ & & 6.44 \\
$\mathrm{~B}_{2} 6$ & 8.15 & $d$ & 8.9 & & 8.34 \\
$\beta_{2}$ & 4.67 & $d$ & 12.2 & & 4.79 \\
$\mathrm{~A}_{1} 2$ & 7.15 & $d d$ & $19.5,8.4$ & $\mathrm{~A}_{1} \mathrm{H}_{3}$ & 7.26 \\
$\mathrm{~A}_{1} 3$ & 6.55 & $d$ & 3.1 & & 6.65 \\
$\mathrm{~A}_{1} 5$ & 6.60 & $d$ & 8.5 & $\mathrm{~A}_{1} \mathrm{H}_{6}$ & 6.65 \\
$\mathrm{~A}_{1} 6$ & 7.15 & $d d$ & $19.5,8.4$ & & 7.26 \\
$\mathrm{~A}_{2} 2$ & 7.15 & $d d$ & $19.5,8.4$ & $\mathrm{~A}_{2} \mathrm{H}_{3}$ & 7.26 \\
$\mathrm{~A}_{2} 3$ & 6.60 & $d d$ & $9.0,2.3$ & & 6.61 \\
$\mathrm{~A}_{2} 5$ & 6.60 & $d d$ & $9.0,2.3$ & & 6.61 \\
$\mathrm{~A}_{2} 6$ & 7.15 & $d d$ & $19.5,8.4$ & & 7.26 \\
\hline
\end{tabular}

Note: Literature data (400 $\left.\mathrm{MHz} \mathrm{CD}_{3} \mathrm{OD}\right)$ derived from Ghogomu et al. (1987)

Table 5. ${ }^{13} \mathrm{C}$ NMR (150 MHz, $\left.\mathrm{CD}_{3} \mathrm{OD}\right), \mathrm{DEPT}, \mathrm{HSQC}$ and HMBC of 18

\begin{tabular}{llllll}
\hline Position & $\boldsymbol{\delta}$ Obs & DEPT & HSQC $\left(\boldsymbol{\delta}_{\mathbf{H}}\right)$ & HMBC & $\boldsymbol{\delta}$ Lit. \\
\hline$\beta_{1}$ & 157.4 & $\mathrm{CH}$ & 8.25 & & 156.4 \\
$\alpha_{1}$ & 122.6 & $\mathrm{C}$ & & $\alpha_{2} \mathrm{H}$ & 122.1 \\
$\mathrm{C}_{1}$ & 177.1 & $\mathrm{C}$ & & $\alpha_{2} \mathrm{H}$ & 175.4 \\
$\mathrm{~B}_{1} 1$ & 117.0 & $\mathrm{C}$ & & & 117.2 \\
$\mathrm{~B}_{1} 2$ & 159.2 & $\mathrm{C}$ & & $\mathrm{B}_{1} \mathrm{H}_{2}$ & 158.5 \\
$\mathrm{~B}_{13}$ & 116.0 & $\mathrm{CH}$ & 6.85 & & 115.9 \\
$\mathrm{~B}_{1} 4$ & 164.7 & $\mathrm{C}$ & & $\mathrm{B}_{1} \mathrm{H}_{2}$ & 163.4 \\
$\mathrm{~B}_{1} 5$ & 103.3 & $\mathrm{CH}$ & 6.72 & & 103.2 \\
$\mathrm{~B}_{16}$ & 128.2 & $\mathrm{CH}$ & 7.89 & & 128.2 \\
$\alpha_{2}$ & 44.7 & $\mathrm{CH}$ & 6.01 & $\beta_{2} \mathrm{H}$ & 43.9 \\
$\mathrm{C}_{2}$ & 204.6 & $\mathrm{C}$ & & $\alpha_{2} \mathrm{H}$ & 204.5 \\
$\mathrm{~B}_{2} 1$ & 114.3 & $\mathrm{C}$ & & & 114.1 \\
$\mathrm{~B}_{2} 2$ & 166.9 & $\mathrm{C}$ & & & 166.8 \\
$\mathrm{~B}_{2} 3$ & 103.5 & $\mathrm{CH}$ & 6.14 & & 103.5 \\
$\mathrm{~B}_{2} 4$ & 166.8 & $\mathrm{C}$ & & & 166.1 \\
$\mathrm{~B}_{2} 5$ & 109.2 & $\mathrm{CH}$ & 6.34 & & 109.0 \\
$\mathrm{~B}_{2} 6$ & 134.4 & $\mathrm{CH}$ & 8.15 & & 134.4 \\
$\beta_{2}$ & 54.3 & $\mathrm{CH}$ & 4.67 & $\alpha_{2} \mathrm{H}$ & 53.4 \\
$\mathrm{~A}_{2} 1$ & 134.9 & $\mathrm{C}$ & & & 134.6 \\
$\mathrm{~A}_{2} 2$ & 130.5 & $\mathrm{CH}$ & 7.15 & & 130.0 \\
$\mathrm{~A}_{2} 3$ & 116.1 & $\mathrm{CH}$ & 6.60 & & 115.8 \\
$\mathrm{~A}_{2} 4$ & 156.7 & $\mathrm{C}$ & & & 156.4 \\
$\mathrm{~A}_{2} 5$ & 116.1 & $\mathrm{CH}$ & 6.60 & & 115.8 \\
$\mathrm{~A}_{2} 6$ & 130.5 & $\mathrm{CH}$ & 7.15 & & 130.0 \\
$\mathrm{~A}_{1} 1$ & 129.7 & $\mathrm{C}$ & & $\mathrm{A}_{1} \mathrm{H}_{3}, \mathrm{~A}_{1} \mathrm{H}_{5}$ & 129.6 \\
$\mathrm{~A}_{1} 2$ & 129.8 & $\mathrm{CH}$ & 7.15 & $\mathrm{~A}_{1} \mathrm{H}_{6}$ & 129.4 \\
$\mathrm{~A}_{1} 3$ & 116.1 & $\mathrm{CH}$ & 6.55 & & 115.9 \\
$\mathrm{~A}_{1} 4$ & 156.7 & $\mathrm{C}$ & & \multirow{2}{*}{$\mathrm{A}_{1} \mathrm{H}_{6}$} & 156.5 \\
$\mathrm{~A}_{1} 5$ & 116.5 & $\mathrm{CH}$ & 6.60 & & 115.9 \\
$\mathrm{~A}_{1} 6$ & 129.8 & $\mathrm{CH}$ & 7.15 & & 129.4 \\
\hline $\mathrm{N}_{1}$ & & & & \\
\hline
\end{tabular}

Note: Literature data derived from Ghogomu et al. (1987); Pegnyemb et al. (2003); Anuradha et al. (2006)

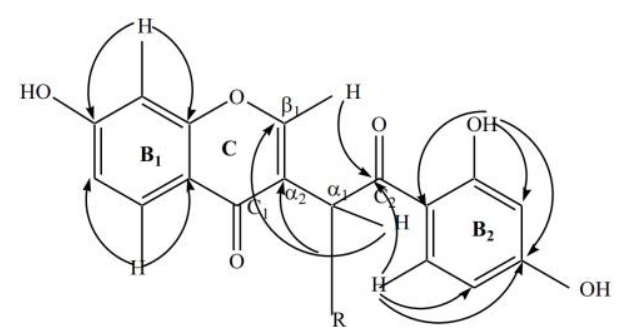

Figure 4. HMBC correlations in the partial structure compound 18

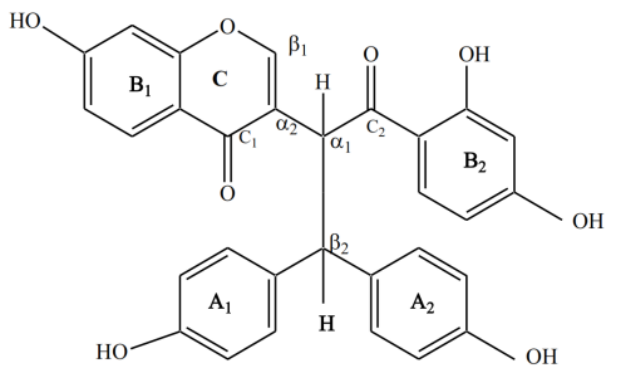

Figure 5. Structure for Lophirone A (18)

\section{Compound 20}

Compound 20 was identified as an amorphous cream white powder from $\mathrm{MeOH}$ soluble fraction of EtOAc root bark. It gave a red color when sprayed with $p$-anisaldehyde and heated to a temperature of $110{ }^{\circ} \mathrm{C}$ for 5 to 10 minutes and fluoresced under UV-light $(254 \mathrm{~nm})$ suggesting the structure of a biflavonoid. Its $\mathrm{mp}$ was $172-176^{\circ} \mathrm{C}$.

The IR spectrum $(\mathrm{KBr}), \mathrm{V}_{\max } \mathrm{cm}^{-1}$ comprised of absorption bands at $3026 \mathrm{~cm}^{-1}$ (C-H aromatic ring str), 957 $\mathrm{cm}^{-1}$ (out of plane bend), $1510 \mathrm{~cm}^{-1}$ and $1452 \mathrm{~cm}^{-1}$ (aromatic $\mathrm{C}=\mathrm{C}-\mathrm{C}$ str). Absence of absorption band at 1620 $\mathrm{cm}^{-1}$ to $1670 \mathrm{~cm}^{-1}$ suggests compound as a chalconoid or an isoflavonoid (Mabry et al. 1970; Peng et al. 2006). The IR spectrum showed a broad O-H absorption band at $3256 \mathrm{~cm}^{-}$ ${ }^{1}$ and aromatic ring stretch at $1611 \mathrm{~cm}^{-1}$. It also exhibited an $\mathrm{OCH}_{3}$ group at $2841 \mathrm{~cm}^{-1}$ and vicinal $\mathrm{C}-\mathrm{H}$ bands at $2715 \mathrm{~cm}^{-1}$. The IR spectrum of 20 and 18 are similar in almost all aspects.

The ${ }^{1} \mathrm{H}-\mathrm{NMR}$ spectrum exhibited signals typical of a 1,2,4-trisubstituted benzene ring representing B1H6 as indicated by a set of the meta-coupled proton at $\delta 6.8, \delta$ 6.66 and an ortho-coupled proton downfield at $\delta 7.86$ (Mabry et al. 1970). The proton resonance which seen as a singlet further downfield at $\delta 8.22$ presumably due to the influence of a keto group suggests an $\mathrm{H}-\beta_{1}$ proton as in an isoflavone system (Mabry et al. 1970; Pegnyemb et al. 2003). 1H NMR resonances represent another set of the $1,2,4$-trisubstituted benzene ring system at $\delta 6.2, \delta 6.43$, and an ortho-coupled proton downfield at $\delta 7.74$ (Pegnyemb et al. 2003a; Anuradha et al. 2006). This ring substructure is near a carbonyl group similar to Lophirone A (18). The ${ }^{1} \mathrm{H}$ NMR spectrum also appeared as two closely para overlapping 1,4-disubstituted benzene rings. The protons integrated for 8 hydrogens and these 2 systems ( $\mathrm{AA}^{\mathrm{C}} \mathrm{XX}$ ') comprise of ortho-coupled protons at $\delta 7.21$, assignable to $\mathrm{A}_{1} \mathrm{H}_{2}$ and $\mathrm{A}_{1} \mathrm{H}_{6}$ and $\delta 6.60$ assignable to $\mathrm{A}_{1} \mathrm{H}_{3}$ and $\mathrm{A}_{1} \mathrm{H}_{5}$ 
as well as $\delta 7.15$ assignable to $\mathrm{A}_{2} \mathrm{H}_{2}$ and $\mathrm{A}_{2} \mathrm{H}_{6}$ and $\delta 6.60$ assignable to $\mathrm{A}_{2} \mathrm{H}_{3}$ and $\mathrm{A}_{2} \mathrm{H}_{5}$ of ring $\mathrm{A}_{2}$, respectively.

The ${ }^{1} \mathrm{H}$ NMR spectrum of compound 20 resembles that of Lophirone A (18) and Afzelone D (19) (Pegnyemb et al. 2003a). It showed the same signal for protons on all the rings present as well as the ring system of two aliphatic protons. Differences observed between compound 18 (Lophirone A) and 20 included modification of the chemical shifts of the ring $\mathrm{A}_{1}, \mathrm{~A}_{2}$, and $\mathrm{B}_{2}$ protons. Also, the presence of two OMe signals at $\delta 3.76$ (a long singlet peak, double integrated, $6 \mathrm{H}$ ) and one OMe signal at $\delta 3.59$ in compound 20 (Table 6).

The double integrated singlet peak suggested that the presence of two symmetrically positioned methoxyl groups, which strongly indicated that compound 20 is a derivative of compounds 18 and 19 (Ghogomu et al. 1987; Messanga et al. 2001; Pegnyemb 2003a; Anuradha et al. 2006; Abdullahi et al. 2014). The ${ }^{13} \mathrm{C}$ NMR spectrum exhibited signals for 33 carbon atoms, including the intensely overlapping signals at $\delta 129.8\left(\mathrm{~A}_{1} \mathrm{C}_{2}\right.$ and $\left.\mathrm{A}_{1} \mathrm{C}_{6}\right)$, $130.5\left(\mathrm{~A}_{2} \mathrm{C}_{2}\right.$ and $\left.\mathrm{A}_{2} \mathrm{C}_{6}\right), 116.1$ (A2C3 and $\left.\mathrm{A} 2 \mathrm{C}_{5}\right)$ and 116.1 (A1C3 and $\mathrm{A}_{1} \mathrm{C}_{5}$ ) (Markham et al. 1978). The carbon chemical shift values displayed 8 of the carbon atoms are oxygenated. The three singlet signals at $\delta 56.1,56.0$ and 55.5 were assignable to methoxy $\left(\mathrm{OCH}_{3}\right)$ group attached to the aromatic ring system. The summary of the $1 \mathrm{D}$ of compound 20 in comparison with $1 \mathrm{D}$ and $2 \mathrm{D}$ results of Lophirone A (18) established and characterized the structure of compound 20 as Afzelone D dimethyl ether (Trimethoxy Lophirone A) (20) (Figure 6).

\section{Compound 17}

Compound 17 was isolated as an amorphous cream white powder from $\mathrm{MeOH}$ soluble fraction of both DCM and EtOAc root bark. It gave a red color when sprayed with $p$ - anisaldehyde and heated to a temperature of $110^{\circ} \mathrm{C}$ for 5 to 10 minutes and fluoresced under UV-light $(254 \mathrm{~nm})$ suggesting the structure of a biflavonoid. The IR spectrum exhibited a broad $\mathrm{O}-\mathrm{H}$ absorption band at $3423 \mathrm{~cm}^{-1}$ and two conjugated carbonyl groups at $1626 \mathrm{~cm}^{1}$ due to aromatic rings and conjugated double bonds. It also presented an $\mathrm{O}-\mathrm{CH}_{3}$ group and vicinal $\mathrm{C}-\mathrm{H}$ absorption bands at 2850 and $2757 \mathrm{~cm}^{-1}$, respectively.

The ${ }^{1} \mathrm{H}$ NMR spectrum of compound 17 was almost similar in many aspects to that of Lophirone A (18), Afzelone D (19) and Afzelone D dimethyl ether (20) (Pegnyemb et al. 2003a). It showed the same signal for protons on all the rings existed as well as the ring system of two aliphatic protons. The only difference noted arises in the modification of the chemical difference of the ring A protons and the existence of one OMe signal at $\delta 3.76$ integrating for $3 \mathrm{H}(\mathrm{s})$ in compound 17 (Table 7). The OMe group can be assigned equally to positions $\mathrm{A}_{1} \mathrm{C}_{4}$ and $\mathrm{A}_{2} \mathrm{C}_{4}$ because its protons are equivalent to those of ring $\mathrm{A}$ of compound 20.

These results strongly suggest that compound 17 is a derivative of Lophirone A and Afzelone D (Ghogomu et al. 1987; Pegnyemb et al. 2003a). The spectral data of compound $\mathrm{MJ} / \mathrm{RE} / \mathrm{OTO}_{2}$ were similar to those reported for Calodenone (17), a compound extracted from the stem bark of Ochna calodendron that is a derivative of lophirone A (Messanga et al. 1992; Pegnyemb et al. 2003a; Anuradha et al. 2006). Compound 17 was therefore recognized to be Calodenone (17) corresponding to the molecular formula $\mathrm{C}_{31} \mathrm{H}_{24} \mathrm{O}_{8}$ and a molecular weight of 524 (Figure 7), and it is the first time to be reported from this plant.

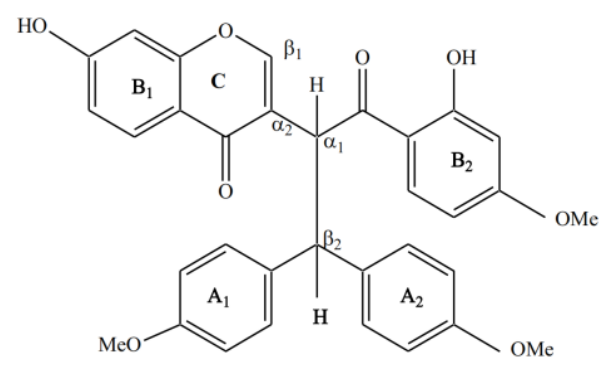

Figure 6. Structure for compound 20

Table 6. ${ }^{1} \mathrm{H}(400 \mathrm{MHz})$ and ${ }^{13} \mathrm{C}$ NMR data for $20(100 \mathrm{MHz}$, $\left.\mathrm{CD}_{3} \mathrm{OD}\right)$

\begin{tabular}{|c|c|c|c|}
\hline Position & $\delta$ Obs & $\delta \mathbf{H}(\mathbf{p p m}), m(J(\mathbf{H z}))$ & $\delta$ Lit. \\
\hline$\overline{\beta_{1}}$ & 157.3 & $8.22(1 \mathrm{H}, s)$ & 156.4 \\
\hline$\alpha_{1}$ & 122.4 & & 122.1 \\
\hline $\mathrm{C}_{1}$ & 177.0 & & 175.4 \\
\hline $\mathrm{B}_{1} 1$ & 117.0 & & 117.2 \\
\hline $\mathrm{B}_{1} 2$ & 128.2 & & 128.2 \\
\hline $\mathrm{B}_{13}$ & 116.0 & $6.82(1 \mathrm{H}, d d, J=2.2 \mathrm{~Hz})$ & 115.9 \\
\hline $\mathrm{B}_{14}$ & 164.7 & & 163.4 \\
\hline $\mathrm{B}_{1} 5$ & 103.3 & $6.66(1 \mathrm{H}, d d, J=2.2,8.6 \mathrm{~Hz})$ & 103.2 \\
\hline $\mathrm{B}_{1} 6$ & 159.4 & $7.86(1 \mathrm{H}, d, J=8.7 \mathrm{~Hz})$ & 158.5 \\
\hline$\alpha_{2}$ & 44.6 & $6.04(1 \mathrm{H}, d, J=12.2 \mathrm{~Hz})$ & 43.9 \\
\hline $\mathrm{C}_{2}$ & 205.2 & & 204.5 \\
\hline $\mathrm{B}_{21}$ & 114.3 & & 114.1 \\
\hline $\mathrm{B}_{2} 2$ & 166.9 & & 166.8 \\
\hline $\mathrm{B}_{2} 3$ & 103.5 & $6.27(2 \mathrm{H}, d, J=8.4 \mathrm{~Hz})$ & 103.5 \\
\hline $\mathrm{B}_{2} 4$ & 166.8 & & 166.1 \\
\hline $\mathrm{B}_{2} 5$ & 109.2 & $6.43(d d, J=2.5 \mathrm{~Hz}, 9.1 \mathrm{~Hz})$ & 109.0 \\
\hline $\mathrm{B}_{2} 6$ & 134.4 & $7.74(4 \mathrm{H}, d, J=9.1 \mathrm{~Hz})$ & 134.4 \\
\hline$\beta_{2}$ & 54.3 & $4.69(2 \mathrm{H}, d, J=12.2 \mathrm{~Hz})$ & 53.4 \\
\hline $\mathrm{A}_{2} 1$ & 134.9 & & 134.6 \\
\hline $\mathrm{A}_{2} 2$ & 130.5 & $7.15(2 \mathrm{H}, d, J=8.4 \mathrm{~Hz})$ & 130.0 \\
\hline $\mathrm{A}_{2} 3$ & 116.1 & $6.56(4 \mathrm{H}, d, J=8.4 \mathrm{~Hz})$ & 115.8 \\
\hline $\mathrm{A}_{2} 4$ & 156.7 & & 156.4 \\
\hline $\mathrm{A}_{2} 5$ & 116.1 & $6.60(4 \mathrm{H}, d, J=8.4 \mathrm{~Hz})$ & 115.8 \\
\hline $\mathrm{A}_{2} 6$ & 130.5 & $7.15(2 \mathrm{H}, d, J=8.4 \mathrm{~Hz})$ & 130.0 \\
\hline $\mathrm{A}_{1} 1$ & 129.7 & & 129.6 \\
\hline $\mathrm{A}_{1} 2$ & 129.8 & $7.21(1 \mathrm{H}, d, J=9.1 \mathrm{~Hz})$ & 129.4 \\
\hline $\mathrm{A}_{1} 3$ & 116.1 & $6.59(1 \mathrm{H}, d, J=2.4 \mathrm{~Hz})$ & 115.9 \\
\hline $\mathrm{A}_{1} 4$ & 156.7 & & 156.5 \\
\hline $\mathrm{A}_{1} 5$ & 116.5 & $6.60(1 \mathrm{H}, d d, J=2.4 \mathrm{~Hz}, 9.1 \mathrm{~Hz})$ & 115.9 \\
\hline $\mathrm{A}_{1} 6$ & 129.8 & $7.21(1 \mathrm{H}, d, J=9.1 \mathrm{~Hz})$ & 129.4 \\
\hline $\mathrm{A}_{1}-4-\mathrm{OCH}_{3}$ & 56.1 & $3.76(3 \mathrm{H}, s)$ & 56.0 \\
\hline $\mathrm{A}_{2}-4-\overline{\mathrm{OCH}}$ & 56.0 & $3.75(3 \mathrm{H}, s)$ & 56.4 \\
\hline $\mathrm{B}_{2}-4-\overline{\mathrm{OCH}_{3}}$ & 55.5 & $3.59(3 \mathrm{H}, s)$ & 55.5 \\
\hline
\end{tabular}

Note: Literature data derived from Messanga et al. (2001); Pegnyemb et al. (2003); Abdullahi et al. (2014) 


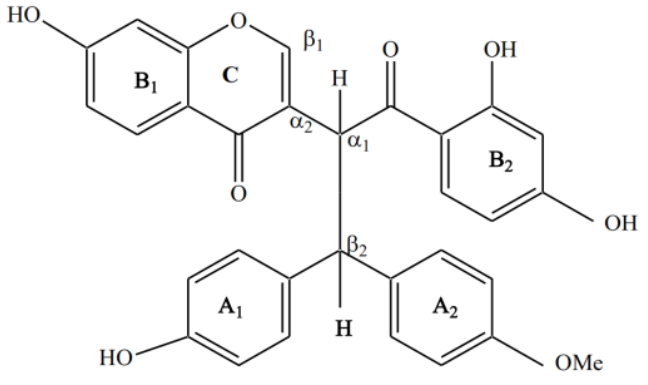

Figure 7. Structure of compound 17

Table 7. ${ }^{1} \mathrm{H}(400 \mathrm{MHz})$ and ${ }^{13} \mathrm{C}$ NMR data for $17(100 \mathrm{MHz}$, $\left.\mathrm{CD}_{3} \mathrm{OD}\right)$

\begin{tabular}{|c|c|c|c|c|}
\hline Position & $\delta$ Obs & $\delta \mathbf{H}(\mathbf{p p m}), \mathbf{m}(\mathbf{J}(\mathbf{H z}))$ & HMBC & $\delta$ Lit. \\
\hline$\overline{\beta_{1}}$ & 157.3 & $8.22(1 \mathrm{H}, s)$ & & 156.4 \\
\hline$\alpha_{1}$ & 122.4 & & $\alpha_{2} \mathrm{H}$ & 122.1 \\
\hline $\mathrm{C}_{1}$ & 177.1 & & $\alpha_{2} \mathrm{H}$ & 175.4 \\
\hline $\mathrm{B}_{1} 1$ & 117.0 & & & 117.2 \\
\hline $\mathrm{B}_{1} 2$ & 128.2 & $7.87(1 \mathrm{H}, d, J=8.7 \mathrm{~Hz})$ & & 128.2 \\
\hline $\mathrm{B}_{1} 3$ & 116.0 & $6.83(1 \mathrm{H}, d d, J=2.1 \mathrm{~Hz})$ & & 115.9 \\
\hline $\mathrm{B}_{14}$ & 164.7 & & $\mathrm{~B}_{1} \mathrm{H}_{2}$ & 163.4 \\
\hline $\mathrm{B}_{1} 5$ & 103.3 & $6.72(1 \mathrm{H}, d d, J=2.1,8.8 \mathrm{~Hz})$ & & 103.2 \\
\hline $\mathrm{B}_{1} 6$ & 159.4 & & $\mathrm{~B}_{1} \mathrm{H}_{2}$ & 158.5 \\
\hline$\alpha_{2}$ & 44.6 & $6.03(1 \mathrm{H}, d, J=12.2 \mathrm{~Hz})$ & $\beta_{2} \mathrm{H}$ & 43.9 \\
\hline $\mathrm{C}_{2}$ & 205.3 & & $\alpha_{2} \mathrm{H}$ & 204.5 \\
\hline $\mathrm{B}_{21}$ & 114.3 & & & 114.1 \\
\hline $\mathrm{B}_{2} 2$ & 166.9 & & & 166.8 \\
\hline $\mathrm{B}_{2} 3$ & 103.5 & $6.14(2 \mathrm{H}, d, J=8.4 \mathrm{~Hz})$ & & 103.5 \\
\hline $\mathrm{B}_{2} 4$ & 166.8 & & & 166.1 \\
\hline $\mathrm{B}_{2} 5$ & 109.2 & $6.29(d, J=2.1 \mathrm{~Hz})$ & & 109.0 \\
\hline $\mathrm{B}_{2} 6$ & 134.4 & $8.21(4 \mathrm{H}, d, J=9.0 \mathrm{~Hz})$ & & 134.4 \\
\hline$\beta_{2}$ & 54.3 & $4.67(1 \mathrm{H}, d, J=12.2 \mathrm{~Hz})$ & $\alpha_{2} \mathrm{H}$ & 53.4 \\
\hline $\mathrm{A}_{2} 1$ & 134.9 & & & 134.6 \\
\hline $\mathrm{A}_{2} 2$ & 130.5 & $7.15(2 \mathrm{H}, \mathrm{d}, \mathrm{J}=8.4 \mathrm{~Hz})$ & & 130.0 \\
\hline $\mathrm{A}_{2} 3$ & 116.1 & $6.58(4 \mathrm{H}, \mathrm{d}, \mathrm{J}=8.4 \mathrm{~Hz})$ & & 115.8 \\
\hline $\mathrm{A}_{2} 4$ & 156.7 & & & 156.4 \\
\hline $\mathrm{A}_{2} 5$ & 116.1 & $6.60(4 \mathrm{H}, \mathrm{d}, \mathrm{J}=8.4 \mathrm{~Hz})$ & & 115.8 \\
\hline $\mathrm{A}_{2} 6$ & 130.5 & $7.15(2 \mathrm{H}, \mathrm{d}, \mathrm{J}=8.4 \mathrm{~Hz})$ & & 130.0 \\
\hline $\mathrm{A}_{1} 1$ & 129.7 & & $\mathrm{~A}_{1} \mathrm{H}_{3}, \mathrm{~A}_{1} \mathrm{H}$ & 5129.6 \\
\hline $\mathrm{A}_{1} 2$ & 129.8 & 7.15 & $\mathrm{~A}_{1} \mathrm{H}_{6}$ & 129.4 \\
\hline $\mathrm{A}_{1} 3$ & 116.1 & $6.55(1 \mathrm{H}, \mathrm{d}, \mathrm{J}=2.4 \mathrm{~Hz})$ & & 115.9 \\
\hline $\mathrm{A}_{1} 4$ & 156.7 & & $\mathrm{~A}_{1} \mathrm{H}_{6}$ & 156.5 \\
\hline $\mathrm{A}_{1} 5$ & 116.5 & $6.60(1 \mathrm{H}, \mathrm{dd}, \mathrm{J}=2.4,9.1 \mathrm{~Hz})$ & & 115.9 \\
\hline $\mathrm{A}_{1} 6$ & 129.8 & $7.15(1 \mathrm{H}, \mathrm{d}, \mathrm{J}=9.1 \mathrm{~Hz})$ & & 129.4 \\
\hline \multicolumn{2}{|c|}{$\mathrm{A}_{1} 4-\mathrm{OCH}_{3} 56.1$} & $3.76(3 \mathrm{H}, \mathrm{s})$ & & 56.0 \\
\hline
\end{tabular}

\section{Compound 23 and 74}

This compound was characterized as white amorphous solid in $\mathrm{CHCl} 3$ soluble fraction of DCM root bark with a melting point $130-134^{\circ} \mathrm{C}$. It gave purple color on spraying in p-anisaldehyde and did not fluoresce in UV suggesting that the compound had steroidal structure. The mass spectra showed a molecular ion peak at $\mathrm{m} / \mathrm{z} 415[\mathrm{M}+\mathrm{H}]+$ and $\mathrm{m} / \mathrm{z} 413[\mathrm{M}+\mathrm{H}]+$ which corresponded to the molecular formula $\mathrm{C} 29 \mathrm{H} 50 \mathrm{O}$ and $\mathrm{C} 29 \mathrm{H} 48 \mathrm{O}$, respectively. The other obvious fragments showed at $\mathrm{m} / \mathrm{z} 396,351,300,271$, and 255 On subjecting to IR spectroscopic analysis, the observed absorption bands are $3459.1 \mathrm{~cm}-1$ that is characteristic of O-H stretching and tri-substituted double bonds. Absorption at $2939.3 \mathrm{~cm}-1$ and $2734.9 \mathrm{~cm}-1$ is due aliphatic $\mathrm{C}-\mathrm{H}$ stretching. Other absorption frequencies include $1647.1 \mathrm{~cm}^{-1}$; as a result of $\mathrm{C}=\mathrm{C}$ stretching; however, this band is weak. At $1463.9 \mathrm{~cm}^{-1}$ is a bending frequency for cyclic $(\mathrm{CH} 2) \mathrm{n}$ and $1381.6 \mathrm{~cm}^{-1}$ is for $\mathrm{CH}_{2}\left(\mathrm{CH}_{3}\right)_{2}$. The absorption frequency at $1053.1 \mathrm{~cm}^{-1}$ signifies cycloalkane. The out of plane $\mathrm{C}-\mathrm{H}$ vibration of the unsaturated part was observed at $881.4 \mathrm{~cm}^{-1}$.

The $1 \mathrm{H}$ NMR of compounds 23 and 74 revealed signals for two singlet methyls at $\delta 1.01$ and 0.68 , four doublet methyls at $\delta 1.02,0.85,0.81$ and 0.8 . There were three vinylic proton signals at $\delta 5.3,5.15$ and 5.02 and an $\mathrm{OH}$ proton signal at $\delta 3.5$. The remaining proton signals were at $\delta$ 0.8-2.4. Direct comparison of the 13C NMR data of 23 and 74 with those reported in the literature (Kovganko et al. 1999; Subhadhirasakul and Pechpongs 2005; Kamboj and Saluja 2011) (Table 9) exhibited they were identical. Thus, 23 and 74 was identified as a mixture of two identical compounds. From the $1 \mathrm{H}$ NMR spectra, integration of proton signals at $\delta 5.3,5.15,5.02$ and 3.53 were in the ratio $2: 1: 1: 2$. Thus, compounds 23 and 74 was confirmed to be a mixture of two of $\beta$-sitosterol (23) and Stigmasterol (74) (Figures 8.A and 8.B, respectively) and approximately in the ratio $1: 1$.

The 13C NMR spectrum showed a total of 47 carbon signals, among them four olefinic carbon signals and one monoxygenated carbon signal was observed. The13C NMR gave a signal at 140.8 and 121.7 ppm for C5=C6 double bond and 138.3 and 129.3 ppm for C22=C23 double bond, respectively for compound 74 and at 140.8 and $121.7 \mathrm{ppm}$ for $\mathrm{C} 5=\mathrm{C} 6$ double bond for compound 23 (Table 8) which demonstrated a high level of saturation in the carbonproton linkages in its structures. The remaining carbons displayed signals having chemical shifts between 11 and 57 ppm. From the above spectroscopic data, it appeared to be a mixture of almost related compounds.

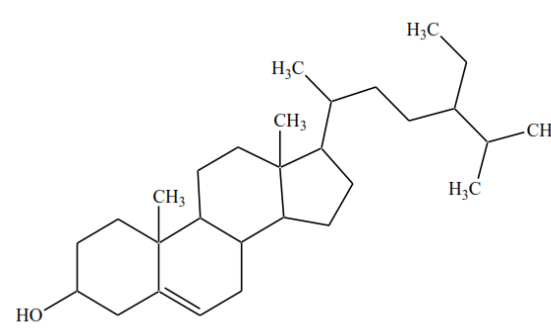

A<smiles>CCC(/C=C/C(C)C1CCC2C3CC=C4CC(C)CCC4(C)C3CCC12C)C(C)C</smiles>

B

Figure 8. A. $\beta$-sitosterol (23), B. Stigmasterol (74) 
Table 8. 13C NMR (100 MHz, CDCl3), of compounds 23 and 74

\begin{tabular}{|c|c|c|c|c|c|}
\hline \multicolumn{2}{|c|}{ Position Group } & \multicolumn{2}{|c|}{ 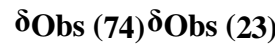 } & \multicolumn{2}{|c|}{$\delta$ Lit. $(74 ; 23)^{M a j o r} \delta^{1} \mathbf{H}$} \\
\hline$\overline{1}$ & $\mathrm{CH}_{2}$ & 37.2 & 37.2 & 37.3 & \\
\hline 2 & $\mathrm{CH}_{2}$ & 31.7 & 31.7 & 31.6 & \\
\hline 3 & $\mathrm{CH}$ & 71.8 & 71.8 & 71.8 & $3.53\left(\mathrm{~m}, \mathrm{H}_{3}\right)$ \\
\hline 4 & $\mathrm{CH}_{2}$ & 42.3 & 42.3 & 42.3 & \\
\hline 5 & $\mathrm{C}$ & 140.8 & 140.8 & 140.1 & \\
\hline 6 & $\mathrm{CH}$ & 121.7 & 121.7 & 121.7 & $5.30\left(\mathrm{~d}, \mathrm{H}_{6}\right)$ \\
\hline 7 & $\mathrm{CH}_{2}$ & 31.7 & 31.7 & $31.9,31.6$ & \\
\hline 8 & $\mathrm{CH}$ & 31.9 & 31.9 & 31.9 & \\
\hline 9 & $\mathrm{CH}$ & 50.2 & 50.2 & $50.1,50.1$ & \\
\hline 10 & $\mathrm{C}$ & 30.5 & 30.5 & 30.5 & \\
\hline 11 & $\mathrm{CH}_{2}$ & 21.1 & 21.1 & 21.1 & \\
\hline 12 & $\mathrm{CH}_{2}$ & 39.7 & 39.8 & $39.7,39.8$ & \\
\hline 13 & $\mathrm{C}$ & 42.3 & 42.3 & 42.3 & \\
\hline 14 & $\mathrm{CH}$ & 56.8 & 56.9 & $56.7,56.9$ & \\
\hline 15 & $\mathrm{CH}$ & 24.3 & 24.3 & $24.3,24.3$ & \\
\hline 16 & $\mathrm{CH}_{2}$ & 28.7 & 28.2 & $28.7,28.2$ & \\
\hline 17 & $\mathrm{CH}$ & 56.1 & 56.1 & $56.7,56.1$ & \\
\hline 18 & $\mathrm{CH}_{3}$ & 11.9 & 12.0 & $11.8,12.0$ & $0.68 \mathrm{CH}_{3}$ \\
\hline 19 & $\mathrm{CH}_{3}$ & 19.4 & 19.4 & 19.4 & $1.01 \mathrm{CH}_{3}$ \\
\hline 20 & $\mathrm{CH}$ & 40.5 & 36.2 & $40.5,36.1$ & \\
\hline 21 & $\mathrm{CH}_{3}$ & 21.1 & 18.8 & $21.1,18.8$ & $1.02 \mathrm{CH}_{3}$ \\
\hline 22 & $\mathrm{CH}, \mathrm{CH}_{2}$ & 138.3 & 33.7 & $137.3,33.7$ & 5.15 \\
\hline 23 & $\mathrm{CH}, \mathrm{CH}_{2}$ & 129.3 & 26.1 & $129.3,26.1$ & 5.02 \\
\hline 24 & $\mathrm{CH}$ & 51.2 & 45.8 & $51.2,45.8$ & \\
\hline 25 & $\mathrm{CH}_{2}$ & 31.9 & 29.2 & $31.9,29.2$ & \\
\hline 26 & $\mathrm{CH}_{3}$ & 20.0 & 19.8 & $19.0,19.8$ & $0.85 \mathrm{CH}_{3}$ \\
\hline 27 & $\mathrm{CH}_{2}$ & 21.2 & 19.0 & $21.2,19.0$ & \\
\hline 28 & $\mathrm{CH}_{3}$ & 25.4 & 23.1 & $25.4,23.1$ & $0.81 \mathrm{CH}_{3}$ \\
\hline 29 & $\mathrm{CH}_{3}$ & 12.0 & 11.7 & $12.0,12.0$ & $0.86 \mathrm{CH}_{3}$ \\
\hline
\end{tabular}

Note: Literature data derived from Kovganko et al. (1999); Subhadhirasakul and Pechpongs (2005)

Table 9. 13C NMR (150 MHz, CDCl3), of compound 75

\begin{tabular}{llllllll}
\hline \multicolumn{2}{l}{ Position Group } & $\boldsymbol{\delta}$ Obs & \multicolumn{3}{l}{$\boldsymbol{\delta}$ Lit. } & \multicolumn{4}{l}{ Position } & Group & $\boldsymbol{\delta}$ Obs & $\boldsymbol{\delta}$ Lit. \\
\hline 1 & $\mathrm{CH}_{2}$ & 15.8 & 15.8 & 18 & $\mathrm{CH}$ & 42.8 & 42.9 \\
2 & $\mathrm{CH}_{2}$ & 35.2 & 35.2 & 19 & $\mathrm{CH}_{2}$ & 35.3 & 35.6 \\
3 & $\mathrm{CH}$ & 72.8 & 72.8 & 20 & $\mathrm{C}$ & 28.2 & 28.2 \\
4 & $\mathrm{CH}$ & 49.2 & 49.1 & 21 & $\mathrm{CH}_{2}$ & 32.8 & 32.9 \\
5 & $\mathrm{C}$ & 60.4 & 59.0 & 22 & $\mathrm{CH}_{2}$ & 39.3 & 39.3 \\
6 & $\mathrm{CH}_{2}$ & 42.8 & 41.8 & 23 & $\mathrm{CH}_{3}$ & 11.6 & 11.6 \\
7 & $\mathrm{CH}_{2}$ & 17.5 & 17.5 & 24 & $\mathrm{CH}_{3}$ & 16.4 & 16.4 \\
8 & $\mathrm{CH}$ & 53.2 & 53.1 & 25 & $\mathrm{CH}_{3}$ & 18.2 & 18.2 \\
9 & $\mathrm{C}$ & 37.8 & 37.8 & 26 & $\mathrm{CH}_{3}$ & 18.7 & 18.6 \\
10 & $\mathrm{CH}$ & 61.3 & 61.3 & 27 & $\mathrm{CH}_{3}$ & 20.1 & 20.1 \\
11 & $\mathrm{CH}$ & 35.6 & 35.6 & 28 & $\mathrm{CH}_{3}$ & 32.1 & 32.1 \\
12 & $\mathrm{CH}$ & 32.8 & 32.9 & 29 & $\mathrm{CH}_{2}$ & 31.8 & 31.8 \\
13 & $\mathrm{C}$ & 38.3 & 38.2 & 30 & $\mathrm{CH}_{3}$ & 35.0 & 35.0 \\
14 & $\mathrm{C}$ & 39.7 & 39.7 & 31 & $\mathrm{CH}_{2}$ & 14.2 & 14.1 \\
15 & $\mathrm{CH}_{2}$ & 30.6 & 30.6 & 32 & $\mathrm{CH}_{3}$ & 37.1 & 37.1 \\
16 & $\mathrm{CH}_{2}$ & 36.1 & 36.1 & $\mathrm{OCOCH}_{3}-$ & 171.2 & 171.1 \\
17 & $\mathrm{C}$ & 30.0 & 29.3 & \multicolumn{2}{l}{$\mathrm{OCOCH}_{3}-$} & 21.1 & 21.4
\end{tabular}

Note: Assignments according to Aragao et al. (1990); Costa and Carvalho (2003)

\section{Compound 75}

This compound was extracted as white amorphous solid in $\mathrm{CHCl} 3$ soluble fraction of DCM root bark with a melting point $266-267^{\circ} \mathrm{C}$. It gave purple color on spraying in $\mathrm{p}$ anisaldehyde and did not fluoresce in UV suggesting that the compound had a triterpenoid skeleton. The UV in $\mathrm{CDCl} 3$ showed one absorption band at $231.5 \mathrm{~nm}$ suggestive of a triterpenoid nucleus (Enas et al. 2012). The calculated molecular mass was 500.4, which corresponded to the molecular formula $\mathrm{C} 34 \mathrm{H} 60 \mathrm{O} 2$. IR $(\mathrm{KBr})$ spectrum peak shows an intense C-H stretch at $2936 \mathrm{~cm}^{-1}, 2866 \mathrm{~cm}^{-1}$ indicates the presence of $-\mathrm{CH} 3$ and $-\mathrm{CH} 2, \mathrm{CH}$ antisymmetric/symmetric stretch. The C-O-C stretching frequency was attributed to $1226 \mathrm{~cm}^{-1}$. At $\delta 171.2$, an acetyl group is linked to $\mathrm{C} 3$, supported by the appearance of a peak in the IR spectrum at $1707 \mathrm{~cm}^{-1}$. As a result, ester carbonyl stretch (Houghton and Lian 1986).

Structural determination of 75 was achieved by careful analysis of $1 \mathrm{D}(1 \mathrm{H}, 13 \mathrm{C}$, and DEPT) NMR spectra. The $1 \mathrm{H}$ NMR spectrum of compound 75 appeared as singlet signals for nine methyl groups of a pentacyclic triterpenoid, and a signal at $\delta 2.00$ is characteristic of an acetyl group. The $1 \mathrm{H}$ NMR data of 75 showed methyl protons at $\delta 0.835-0.982$, one $\mathrm{C} 3$ proton at $\delta 3.70$ are typical of ester protons, and $\delta$ 4.08 is characteristic of two methylene hydrogens. The $1 \mathrm{H}$ NMR data did not allow much analysis of the hindered signals. However, we observed a doublet $(\mathrm{J}=7.8 \mathrm{~Hz})$ centered at $\delta 0.835 \mathrm{ppm}$ characteristic of a methyl group at position C23 of friedelane compounds (Lopez-Perez et al. 2007).

Comparing the $1 \mathrm{H}$ and $13 \mathrm{C}$ NMR data of compound 75 with that of $3 \beta, 24$ - diacetylfriedelane (76) implied that compound 75 was similar to 76 in rings $\mathrm{A}, \mathrm{B}, \mathrm{C}$ and $\mathrm{D}$ (Figure 9). It suggests that the acetyl group is bound to $\mathrm{C} 3$, which was supported by the HMBC correlations of the latter (Mahato and Kundu 1994; Carvalho et al. 1995). The DEPT 13C NMR spectra were used to recognize the signals corresponding to six quaternary carbons, one monoxygenated carbon at $\delta 72.8$ besides signals of nine methyls, twelve methylene, six methylene groups and one acetyl group $(\delta 171.2,21.1)$. From the DEPT spectra, the compound has 34 carbons, of which 27 bear proton. Compound 75 was given the name $3 \beta$-acetyl-24ethylfriedelane.

\section{Antibacterial activity test for the isolated compounds}

The activity of isolated compounds 17 to 75 was measured using the same procedure as discussed for the crude extracts, based on the method used by Chhabra and Usio (1991). The inhibition zone was determined as in the crude extracts using the same pathogens except for E. coli. An isolated compound showing a clear area of $9 \mathrm{~mm}$ or higher was considered to have significant activity. The antimicrobial investigations suggest the compounds have activity against $S$. aureus and $S$. typhi, however, were not active at the tested $E$. coli (Table 10). The isolated compounds displaying zones of inhibition below $9 \mathrm{~mm}$ were considered to be inactive (Faizi et al. 2003). All the compounds showed activity against all indicator strains, but compound mixture 23 and 74 had moderate activity as presented (Table 10). 
<smiles>CCC12CCC3C4CC(C)(C)CCC4(C)CCC34CCCC1(C)C4CCC(O)C2C</smiles><smiles>CCC12CCC3C(C)C(O)CCC3C1(C)CCC1(C)CCC(C)(C)CC12C</smiles>

$\mathbf{R}_{1}=\mathrm{Ac}$

$$
\mathbf{R}_{2}=\mathrm{Et}
$$

A

$$
\mathbf{R}_{1}=\mathbf{R}_{2}=A c
$$

B

Figure 9. Structure for A. 75 , B. 76

Table 10. Inhibition zones (in $\mathrm{mm}$ ) of antibacterial activity of isolated compounds

\begin{tabular}{llll}
\hline Compound & S. aureus & E. coli & S. typhi \\
\hline Compound 75 & 16 & 6 & 12 \\
Compound 17 & 12 & 6 & 14 \\
Compound 20 & 14 & 7 & 14 \\
Compound 18 & 18 & 6 & 12 \\
Compound 23 and 74 & 10 & 6 & 6 \\
Chloramphenicol (+ve) & 25 & 24 & 23 \\
DMSO (-ve) & 6 & 6 & 6
\end{tabular}

Note: Staphylococcus aureus ATCC 25724, Escherichia coli ATCC 25723, Salmonella. typhi (clinical isolate)

The study supports the finding that biologically-active compounds and potential pharmaceuticals can be isolated from medicinal plants. As was found from other Ochnaceae species, the majority of the compounds isolated from the plant were biflavonoids. This project aimed at investigating and establishing the presence of antimicrobial activity in Ochna thomasiana based on its use in traditional medicine against microbial infections. The following was found: (i) The plant crude extracts had appreciable yields, especially the $\mathrm{MeOH}$ extracts. (ii) There was a notable activity for the root and stem bark $\mathrm{MeOH}$ extracts with MICs against the tested Gram-positive bacteria fell between 500 and 1000 $\mu \mathrm{g} / \mathrm{mL}$. (iii) The $\mathrm{MeOH}$ root bark extract showed the greatest activity against Gram-positive bacteria (iv). All the extracts had no activity against the Gram-negative bacteria strains. (v) We isolated and characterized three biflavonoids and three sterols. (vi) Compounds 18, 20, and 75 from the plant had fairly noticeable activity against Sa. typhi and S. aureus ranging between 12-18 mm. However, all the isolated compounds had no activity against $E$. coli.

\section{REFERENCES}

Abdullahi MI, Musa AM, Haruna AK, Pateh UU, Sule IM, Abdulmalik IA, Abdullahi MS, Abimiku AG, Iliya I. 2014. Isolation and characterization of an anti-microbial biflavonoid from the chloroform-soluble fraction of methanolic root extract of Ochna schweinfurthiana (Ochnaceae). Afr J Pharmacol 8 (4): 92-99. DOI: 10.5897/AJPP2013.3520.
Abraham RJ, Mobli M, Smith RJ. 2003. ${ }^{1} \mathrm{H}$ chemical shifts in NMR: Part 19. Carbonyl anisotropies and steric effects in aromatic aldehydes and ketones. Magn Reson Chem 41: 26-36. DOI: 10.1002/mrc.1125

Amin A, Mousa M. 2007. Merits of anti-cancer plants from Arabian Gulf region. Cancer Ther 5: 55-56.

Andrews JM. 2001. Determination of minimum inhibitory concentrations. J Antimicrob Chemother 48 (1): 5-16. DOI: 10.1093/jac/48.suppl_1.5

Anuradha V, Srinivas PV, Rao RR, Manjulatha K, Purohit MG, Rao JM. 2006. Isolation and synthesis of analgesic and anti-inflammatory compounds from Ochna squarrosa. Bioorg Med Chem Lett 14: 68206826. DOI: 10.1016/j.bmc.2006.06.048

Aragao Jr AG, Ribeiro RA, magalhaes JG, Vale MR. 1990. Effects of Ageratum conyzoides in nociception and inflammatory response induced by Zymosan. Fitoterapia 61: 349-354.

ASFMT. 2002. Arubuko Sokoke Strategic Forest Management plan 2002 2027. Kenya Forest Research Institute, Nairobi.

Balunas MJ, Kinghorn AD. 2005. Drug discovery from medicinal plants. Life Sci 78: 431-441. DOI: 10.1016/j.lfs.2005.09.012

Bonjar G, Farrokhi PR. 2004. Antibacillus activity of plants used in traditional medicine of Iran. Niger J Nat Prod Med 8: 34-39. DOI: 10.4314/njnpm.v8i1.11811

Bushra BNR, Ganga DT. 2003. Antibacterial activity of selected seaweeds from Kovalam south west coast of India. Asian J Microbiol Biotechnol Environ Sci 5 (3): 319-322.

Carvalho MG, de Almeida MEL, de Hauptli MB, Meleiro LAC. 1995. Triterpenos isolados de Eschweilera rabeliana Mori (Lecythidaceae). Revista. Universidade Rural: Série Ciências Exatas e da Terra 17: $33-$ 36.

Chhabra SC, Uiso FC. 1991. Antibacterial activity of some Tanzania plants, used in traditional medicine. Fitoterapia 62: 499-504.

Costa DaPM, Carvalho DeMG. 2003. Triterpenes and saponin from Eschweilera longipesmori (Lecythidaceae). Ann Braz Acad Sci 75 (1): 21- 25. DOI: 10.1590/S0001-37652003000100003

Cragg GM, Newman DJ. 2005. Plants as source of anticancer agents. J Ethnopharmacol 100: 72-79. DOI: 10.1016/j.jep.2005.05.011

de Boer JH, Kool A, Broberg A, Mziray WR, Hedberg I, Levenfors JJ. 2005. Anti-fungal and anti-bacterial activity of some herbal remedies from Tanzania. J Ethnopharmacol 95: 461-469. DOI: 10.1016/j.jep.2004.09.035

Duraipandiyan V, Ignacimuthu S. 2007. Antibacterial and antifungal activity of Cassia fistulal; An ethnomedicinal plant. J Ethnopharmacol 112: 590-593. DOI: 10.1016/j.jep.2007.04.008

Edeoga HO, Okwu DE, Mbaebie BO. 2005. Phytochemical constituents of some Nigerian medicinal plants. Afr J Biotechnol 4: 685-688. DOI: 10.5897/AJB2005.000-3127

Elgayyar M, Draugho FA, Golden DA, Mont JN. 2000. Antimicrobial activity of essential oils from plants against selected pathogenic and saprophytic microorganisms. J Food Prot 64: 1019-1024. DOI: 10.4315/0362-028X-64.7.1019.

Enas A, Sameh A, Ahmed S. 2012. Phytochemical and biological studies on isoflavonoids from Dalbergia paniculata cultivated in Egypt. Pharmacologia 3: 84-90. DOI: 10.5567/pharmacologia.2012.84.90.

Faizi S, Mughal NR, Khan RA, Khan SA, Ahmed A, Bibi N, Ahmed SA. 2003. Properties of, Polyalthia longifollia var. Pendua: Isolation of a lactone and active antimicrobial agent from the ethanol extract of the stem. Phytother Res 17: 1177-1181. DOI: 10.1002/ptr.1333.

Farnsworth NR, Akerele RO, Bingel AS, Soejarto DD, Guo Z. 1985. Sources, action and uses of plant-derived drugs and their corrections. Bull WHO 63: 965-981.

Fennell CW, Lindsey KL, McGaw LJ, Sparg SG, Stafford GI, Elgorashi EE, Grace OM, Van Staden J. 2004. Assessing african medicinal plants for efficacy and safety: pharmacological screening and toxicology. J Ethnopharmacol 93: 205-217. DOI: 10.1016/j.jep.2004.05.012.

Ghogomu R, Sodengam BL, Martin MT, Bodo B. 1987. Lophirone A, a biflavonoid with unusual skeleton from Lophira lanceolata. Tetrahedron Lett 28 (26): 2967-2968. DOI: 10.1016/S00404039(00)96256-7.

Gurib-Fakim A. 2006. Medicinal plants: traditions of yesterday and drugs of today. Mol Asp Med 27: 1-10. DOI: 10.1016/j.mam.2005.07.008

Houghton PJ, Lian LM. 1986. Triterpenoids from Desfontainia spinosa. Phytochemistry 25 (8): 1939-1944. DOI: 10.1016/S00319422(00)81179-6.

Kamboj A, Sakuja KA. 2011. Isolation of stigmasterol and $\beta$-sitosterol from petroleum ether extract of aerial parts of ageratum conyzoides (Asteraceae). Int J Pharm Pharm Sci 3 (1): 94-96. 
Koehn FE, Carter GT. 2005. The evolving role of natural products in drug discovery. Nat Rev Drug Discov 4: 206-220. DOI: 10.1038/nrd1657.

Kovganko NV, Kashkan ZhN, Borisov EV, Batura EV. 1999. ${ }^{13} \mathrm{C}$ NMR of $\beta$ - sitosterol derivatives with oxidized rings $A$ and $B$. Chem Nat Compd 35: 646-649. DOI: 10.1007/BF02236293.

López-Pérez JL, Therón R, Del Olmo E, Díaz D. 2007. NAPROC-13: a database for the dereplication of natural product mixtures in bioassay guided protocols. Bioinformatics 23: 3256-3257. DOI: 10.1093/bioinformatics/btm516.

Mabry TJ, Markham KR, Thomas MB. 1970. The systematic Identification of Flavonoids. Springer-Verlag, Heidelberg. DOI: 10.1007/978-3-642-88458-0.

Magassouba FB, Diallo A, Kouyaté M, Mara F, Mara O, Bangoura O, Camara A, Traoré S, Diallo AK, Zaoro M, Lamah K. 2007. Ethnobotanical survey and antibacterial activity of some plants used in Guinean traditional medicine. J Ethnopharmacol 114: 44-53. DOI: 10.1016/j.jep.2007.07.009.

Mahato SB, Kundu AP. 1994. ${ }^{13} \mathrm{C}$ NMR Spectra of pentacyclic triterpenoids. A compilation and some salient features. Phytochemistry 37: 1517-1575. DOI: $10.1016 /$ S0031-9422(00)89569

Markham KR, Ternai B, Stanley R, Geiger H, Mabry TJ. $1978 .{ }^{13}$ C-NMR Studies of Flavonoids II. Tetrahedron 34: 1391-1397. DOI: 10.1016/0040-4020(78)88336-7.

McCloud T, Nemee J, Muschik G, Shieffield H, Quesenberry P, Suffness M, Cragg G, Thomson J. 1998. Extraction of bioactive molecules from plants. Presented in Park City, Utah. Int Congr Nat Prod 17-21.

Messanga BB, Ghogomu TR, Sondengam BL, Martin MT, Bodo B. 1992. Calodenone, a new isobiflavonoid from Ochna calodendron. J NatProd 55: 245-257. DOI: 10.1021/np50080a018.

Messanga BB, Sondengam BL, Bodo B. 2001. Calodendroside A: a Taxifolin diglucoside from the stem bark of Ochna calodendron. Can J Chem 78: 487-489. DOI: 10.1139/v00-037

Michael JP, Chan ECH, Noel RK, Merna FP. 2003. Microbiology $5^{\text {th }}$ edition. Tata McGraw-hill Publishing Company Ltd, New Delhi.

Pegnyemb DE, Tih RG, Sondengam BL, Blond A, Bodo B. 2003a Isoflavonoids of Ochna afzelii. Pharm Biol 41: 91-94. DOI 10.1076/phbi.41.2.92.14245.

Pegnyemb DE, Tih RG, Sondengam BL, Blond A, Bodo B. 2003b. Flavonoids of Ochna afzelii. Phytochemistry 64: 661-665. DOI 10.1016/S0031-9422(03)00267-X
Peng JY, Fan GR, Wu YT. 2006. Two new Dihydroflavonoids from Patrinia villosa Juss. China J Chemother 17 (2): 218-220.

Ramawat KG, Goyal S. 2008. The Indian herbal drugs scenario in global perspectives. Bioactive Molecules and Medicinal Plants. Springer, Berlin Heidelberg, New York. DOI: 10.1007/978-3-540-74603-4 18.

Randerath H, Jawetz E, Melnick J, Adelberg E. 1968. Review of medical microbiology $7^{\text {th }}$ edition, Lange Medical Publications, Canada.

Runyoro D, Matee M, Olipa N, Joseph C, Mbwambo H. 2006. Screening of Tanzanian Medicinal Plants for anti-candida activity. BMC Compl Altern Med 6 (11): 1-10. DOI: 10.1186/1472-6882-6-11.

Shahidi BH. 2004. Evaluation of antimicrobial properties of Irananian Medicinal Plants against Micrococcus luteus, Serratia marcescens, Klebsiella pneumonia and Bordetella brochoseptica. Asian J Plant Sci 3: 82-86. DOI: 10.3923/ajps.2004.82.86.

Silverstein RM, Webster FX, Kiemle DJ. 2005. Spectrometric Identification of Organic Compounds. John Wiley and Sons, New York.

Subhadhirasakul S, Pechpongs P. 2005. A terpenoid and two steroids from the flowers of Mammea siamensis, Songklanakarin Thai Herbs. J Sci Technol 27 (2): 555-561.

Tania MAA, Axréia FS, Mitzi B, Telma SMG, Elza AS, Artur SJ, Carlos LZ. 2000. Biological screening of brazilian medicinal plants. Memórias do Instituto Oswaldo Cruz 95 (3): 367-373. DOI: 10.1590/S0074-02762000000300012.

Thangadurai D, Murphy KSR, Prasad PJN, Pullaian T. 2004. Antimicrobial screening of Decapelis hamiltonii Wightad Arn. (Asclepiadaceae) root extracts against food related microorganism. J Food Sci 24: 239-245. DOI: 10.1111/j.1745-4565.2004.00537.x.

Uggerud E, Petrie S, Bohme DK, Turecek F, Schröder D, Schwarz H, Plattner D, Wyttenbach T, Bowers MT, Armentrout PB, Truger SA. 2003. Topics in Current Chemistry: Modern Mass Spectroscopy. Springer-Verlag, Berlin.

Vasavi Y, Parthiban N, Sathis KD, Banji D, Srisutherson N, Somsubhara GM, Chakravarthy VK. 2011. Heteronuclear Multible Bond Correlation Spectroscopy. Int J PharmTech Res 3 (3): 1410-1422.

WHO. 2002. WHO Traditional Medicine Strategy 2002-2005. WHO Geneva.

WHO. 2008. Traditional medicine. Fact sheet No. 134 